\title{
Methods for the synthesis of $\alpha$-heterocyclic/heteroaryl- $\alpha$-aminophosphonic acids and their esters
}

\author{
Tarik E. Ali* and Somaia M. Abdel-Kariem \\ Department of Chemistry, Faculty of Education, Ain Shams University, Roxy, Cairo, Egypt \\ E-mail: tarik_elsayed1975@yahoo.com
}

DOI: http://dx.doi.org/10.3998/ark.5550190.p009.112

\begin{abstract}
This review describes a comprehensive account of methods which are commonly applied for the synthesis of $\alpha$-heterocyclic/heteroaryl $\alpha$-aminophosphonic acids and their esters. In the following order, protocols based on the methodologies listed below are discussed: (a) Pudovik reaction; (b) Kabachnik-Fields reaction and (c) Miscellaneous Methods.
\end{abstract}

Keywords: $\alpha$-Aminophosphonates, heterocycles, Kabachnik-Fields, Pudovik reactions

\section{Table of Contents}

1 Introduction

2 Synthesis by Pudovik Reactions

2.1 Five-membered heterocycles with one heteroatom

2.2 Five-membered heterocycles with two heteroatoms

2.3 Six-membered heterocycles with one heteroatom

3 Synthesis by Kabachnik-Fields Reactions

3.1 Five-membered heterocycles with one heteroatom

3.2 Five-membered heterocycles with two heteroatoms

3.3 Six-membered heterocycles with one heteroatom

3.4 Six-membered heterocycles with two or more heteroatoms

3.5 Macrocycles

4 Miscellaneous Methods

4.1 From diethyl $\alpha$-azido- $\alpha$-(benzoylaminomethyl)phosphonate

4.2 Nucleophilic substitution reactions

4.3 Cycloaddition of $\alpha$-alkylaminophosphonates

4.4 Reduction of $\alpha$-hydroxyiminophosphonate

4.5 Hydrolysis of $S$-adenosyl-L-homocysteine derivative 
4.6 Curtius rearrangement of $\alpha$-acylazidophosphonate

4.7 Addition of diethyl phosphite to chiral $N$-benzylnitrones

4.8 From phosphonyliminium salts

4.9 From oxazolyl phosphonates

5 Conclusions

6 References

\section{Introduction}

$\alpha$-Aminophosphonic acids are considered mimics of the corresponding $\alpha$-aminocarboxylic acid. ${ }^{1}$ The phosphonic moiety has long been established as a bioisostere of a carboxylic unit. These features explain the large range of biological activities displayed by the members of this important class of compounds and the applications. ${ }^{2-5}$ They have been found in areas ranging from medicine to agriculture, for example, as antibiotics, ${ }^{6}$ enzyme inhibitors, ${ }^{7}$ anticancer agents ${ }^{8}$ and herbicides. ${ }^{9,10}$ These biological properties mostly are associated with the tetrahedral structure of the phosphonyl group acting as a "transition-state" analogue. ${ }^{11}$ Because of their ability to mimic transition states of hydrolysis, phosphonic acid derivatives having heterocycles at the $\alpha$-positions have been shown to be inhibitors of various enzymes, including HIV-protease and human collagenase. ${ }^{12}$

At present, the literature concerning the synthesis and application of $\alpha$-aminophosphonates is very extensive, comprising more than six thousand publications. Hence, several approaches ${ }^{13}$ have been developed for the synthesis of $\alpha$-aminophosphonates. Two main pathways are: (i) the Pudovik reaction, where dialkyl phosphites are added to imines, and (ii) the Kabachnik-Fields three component reaction, in which a carbonyl, an amine and a di- or tri-alkyl phosphite react in a single-pot. In some reports, these reactions were carried out as straightforward one-pot procedures without any catalyst, ${ }^{14}$ but in most cases they were performed using catalysts. ${ }^{15}$ On the other hand, $\alpha$-aminophosphonic acids and their esters bearing a heterocyclic moiety at the $\alpha$-position are becoming the subject of growing interest. To our knowledge, there are several methods for the synthesis of $\alpha$-heterocyclic/heteroaryl $\alpha$-aminophosphonates (Figure 1). In connection with our work on the preparation of $\alpha$-aminophosphonates containing heterocyclic systems, ${ }^{16-19}$ we report in this review article all the available synthetic methods of $\alpha$-heterocyclic/heteroaryl $\alpha$-aminophosphonates which were published until 2013.

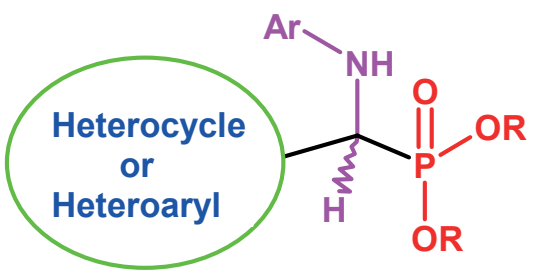

Figure 1. $\alpha$-Heterocyclic/heteroaryl- $\alpha$-aminophosphonic acids and their esters. 


\section{Synthesis by Pudovik Reaction}

\subsection{Five-membered heterocycles with one heteroatom}

The Schiff bases 1 were subjected to react in situ with diethyl phosphite in toluene at $110{ }^{\circ} \mathrm{C}$ to give the corresponding $\alpha$-aminophosphonate esters 2 . When diphenyl phosphite was used in the reaction with imines $\mathbf{1}$, the addition reaction took place even at room temperature, giving the diphenyl esters in high yields (Scheme 1). ${ }^{20}$
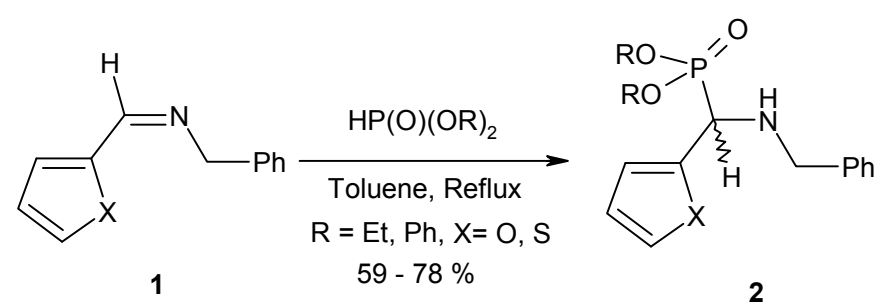

\section{Scheme 1}

Stereoselective synthesis of (5-hydroxymethylfuran-2-yl)- $N$-( $\alpha$-methylbenzylaminophosphonates 4a,b was performed by the addition of dibenzyl phosphite to the $N$-(furylmethylene)-(R)- $\alpha$-methylbenzylamine (3), resulting in diastereoisomeric esters (Scheme 2). ${ }^{21,22}$
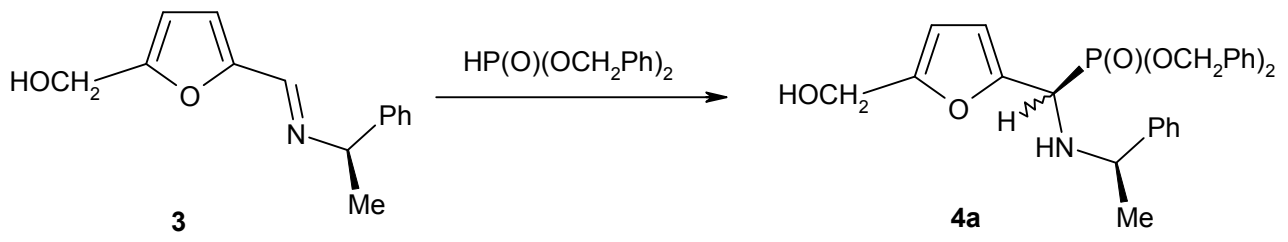

Major product, $77 \%$

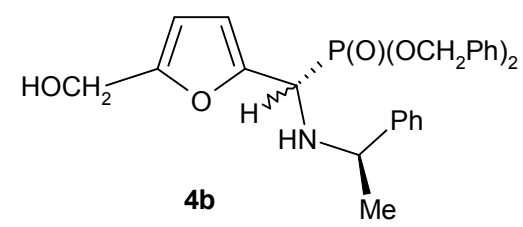

Minor product, $23 \%$

\section{Scheme 2}

When the cyclic phosphonate 5 was reacted with the Schiff base 6 at $17{ }^{\circ} \mathrm{C}$ it afforded the corresponding $\alpha$-(3-thienyl)- $\alpha$-aminophosphonate 7 . Reaction of phosphonate 5 ( $\mathrm{R}=\mathrm{COOR})$ with the imine 6 required ultrasonic conditions to bring the reaction to completion (Scheme 3$){ }^{23}$ 


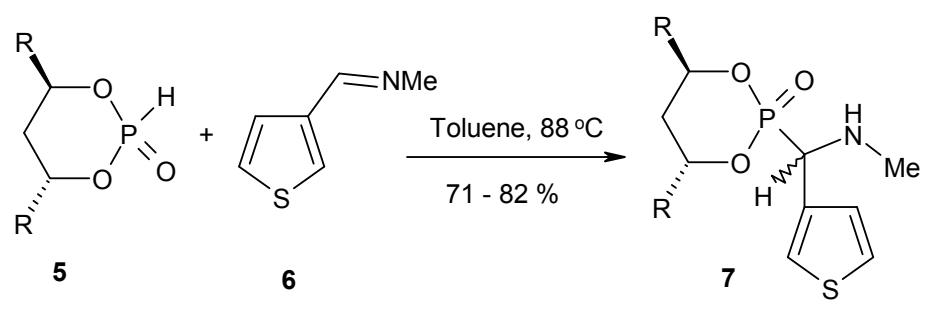

$\mathrm{R}=\mathrm{a}) \mathrm{CH}_{3}$, b) $\left(\mathrm{CH}_{3}\right)_{2}$, d) COOR

\section{Scheme 3}

Reaction of the Schiff bases 8 with dialkyl phosphites in toluene at $85{ }^{\circ} \mathrm{C}$ provided the corresponding $\alpha$-aminophosphonates 9 (Scheme 4). ${ }^{24}$

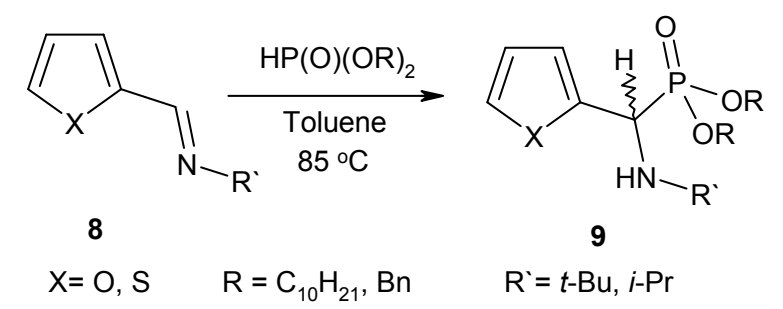

\section{Scheme 4}

The diethyl phosphonate esters 11 were prepared by heating equimolar mixtures of diethyl phosphite and the corresponding Schiff base $\mathbf{1 0}$ in the absence of solvent at temperatures between 90 and $100{ }^{\circ} \mathrm{C}$ (Scheme 5). ${ }^{25}$

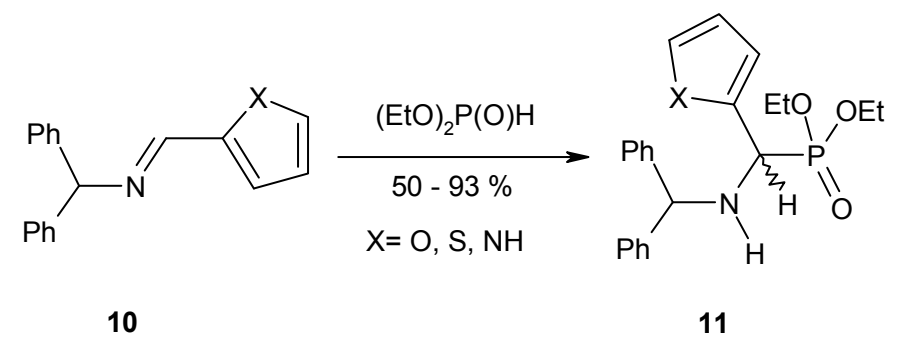

\section{Scheme 5}

Similarly, addition of two equivalents of dimethyl phosphite to heterocyclic imine $\mathbf{1 2}$ in methanol afforded the $\alpha$-(2-furyl)- $\alpha$-aminophosphonate 13 (Scheme 6). ${ }^{26}$ 


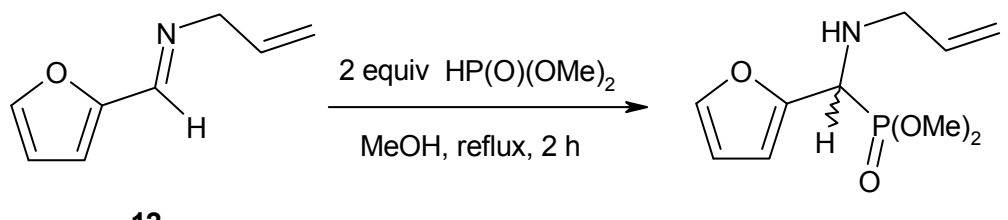

12

13

\section{Scheme 6}

Synthesis of cucurbitine phosphonic analogues $\mathbf{1 6}$ was performed through reaction of hydrazone intermediates 14 with triethyl phosphite in acidic media. Subsequent cleavage of $\mathrm{N}-\mathrm{N}$ bonds gave aminophosphonic acid $\mathbf{1 6}$ and not the corresponding $\alpha$-hydrazinophosphonate (cucurbitine analogue) (Scheme 7). ${ }^{27}$

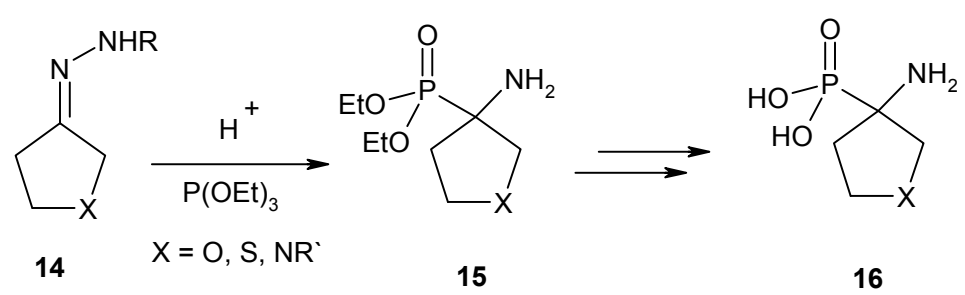

\section{Scheme 7}

4,4'-Bis[[(dialkoxyphosphonyl)-(2-furyl)methyl]amino]diphenyl (18) was prepared by addition of diethyl phosphite to $N, N^{\prime}$-bis(furfurylidene)benzidine (17) in sodium ethoxide and stirring at room temperature for 3 hours (Scheme 8$){ }^{28}$

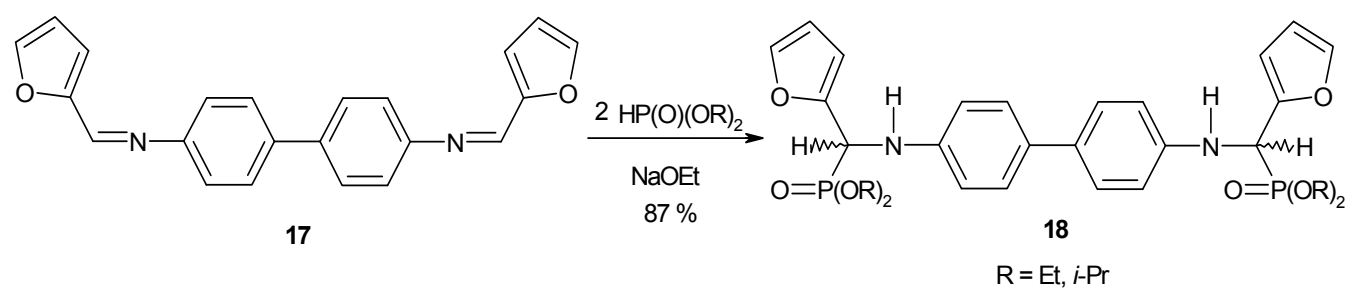

\section{Scheme 8}

Similarly, addition of diethyl phosphite to the azomethine bonds of the bis-Schiff base 19 was carried out, affording 1,3-bis[N-[(diethoxyphosphonyl)-(2-furyl)methyl]amino]benzene (20). In this case NMR studies revealed that the reaction product is a mixture of the two possible diastereomeric forms: $R, S$ (meso) and the enantiomeric pair $R, R$ and $S, S$ (Scheme 9). ${ }^{29}$ 


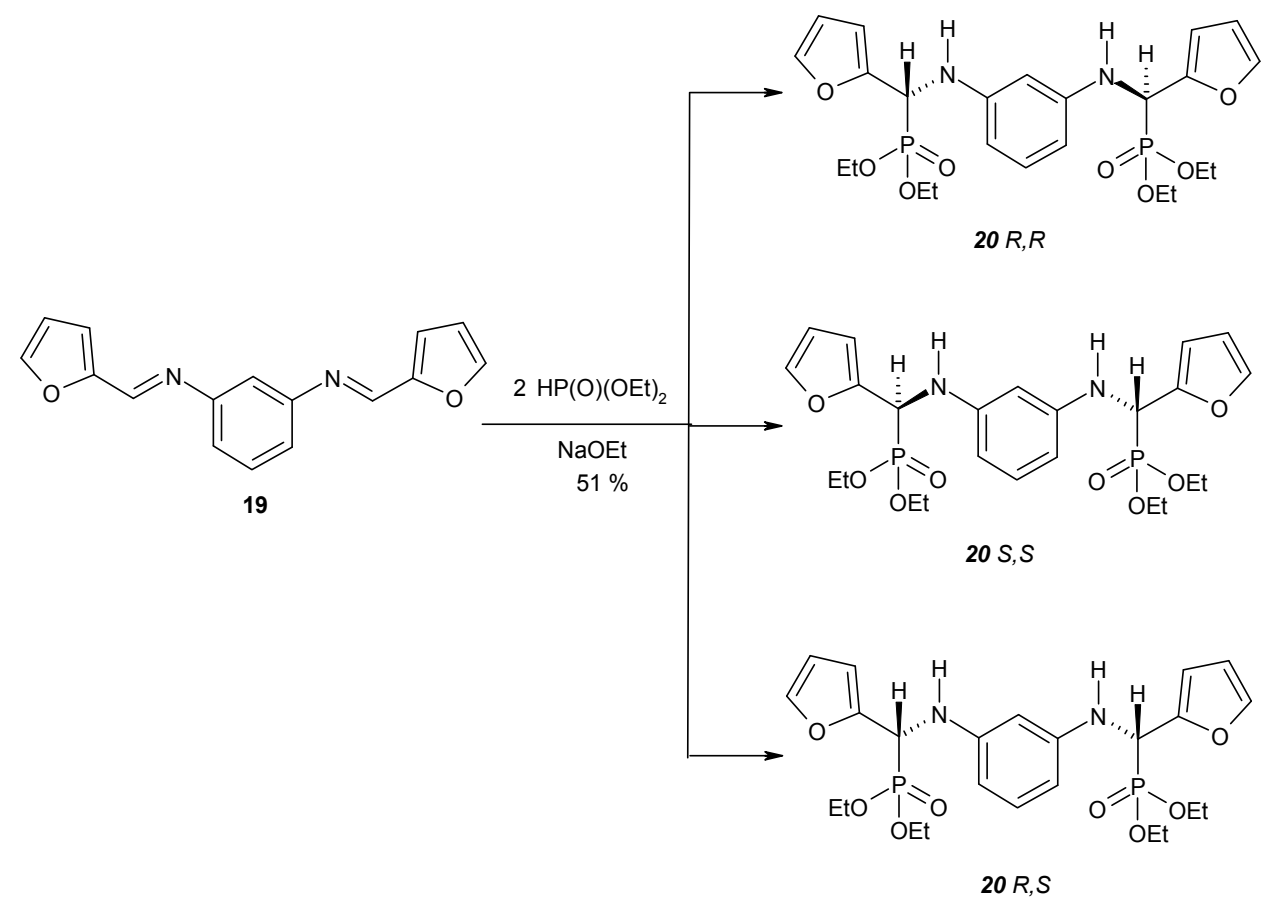

\section{Scheme 9}

Also, four bis(aminophosphonates) 25, 26, 27 and 28 were synthesized through addition of diethyl phosphite to the azomethine bonds of the furan-substituted bis(imines) 21-24. The addition of dialkyl (diaryl) phosphites to bis(imines) should lead to the formation of two diastereomeric forms, meso and racemic diastereomers. Thus, this synthesis in most cases occurs with high stereoselectivity, yielding as major product only one of the diastereomers, as previously obtained in similar reactions (Scheme 10). ${ }^{30,31}$

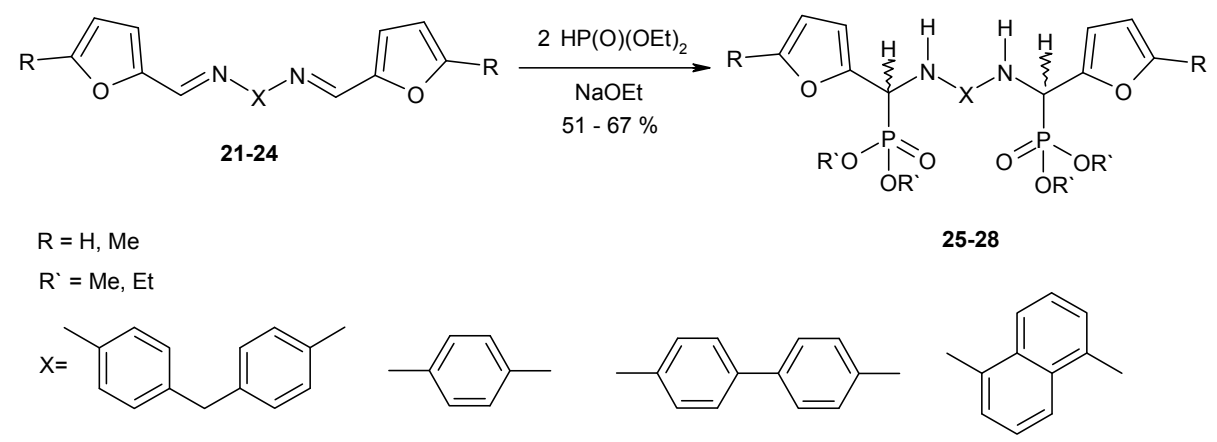

\section{Scheme 10}

Poly(oxyethylene)aminophosphonates $\mathbf{3 1}$ were synthesized through addition of poly (oxyethylene H-phosphonates) 29 to the azomethine bond of $N$-furfurylidene toluidine (30), according to Scheme 11. The polymer analogous reaction was carried out in the presence of 
catalytic $\mathrm{CdI}_{2}$, as well as without catalyst. In the presence of $\mathrm{CdI}_{2}$ the addition of $\mathrm{P}-\mathrm{H}$ groups to the azomethine 30 proceeded with higher reaction rate compared to the non-catalyzed reaction and the poly ( $\alpha$-aminophosphonates) 31 were obtained in good yields in 3 hours. In the absence of catalyst the reaction time was longer, up to 15 hours (Scheme 11). ${ }^{32}$

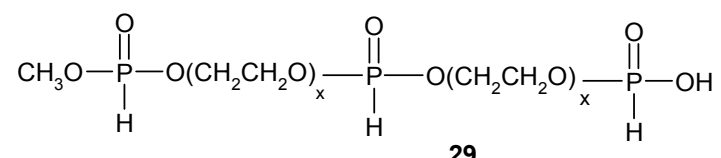

29
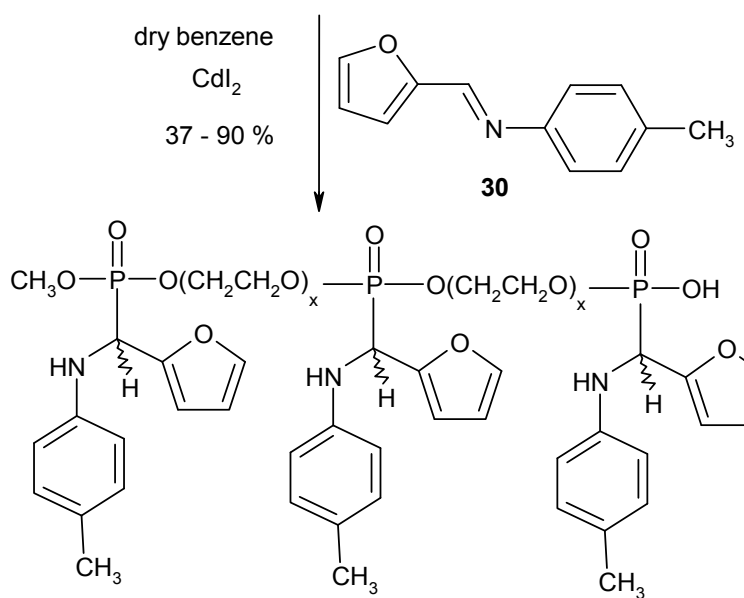

$X=4,13$
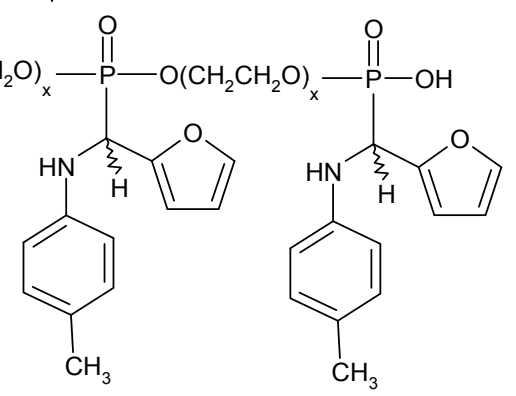

31

\section{Scheme 11}

Reaction of Schiff bases 32 with bis(trimethylsilyl)hydrogen phosphite in boiling benzene gave $\alpha$-(3-indolyl)- $\alpha$-aminophosphonates 33 in yields $90-93 \%$ (Scheme 12). ${ }^{33}$
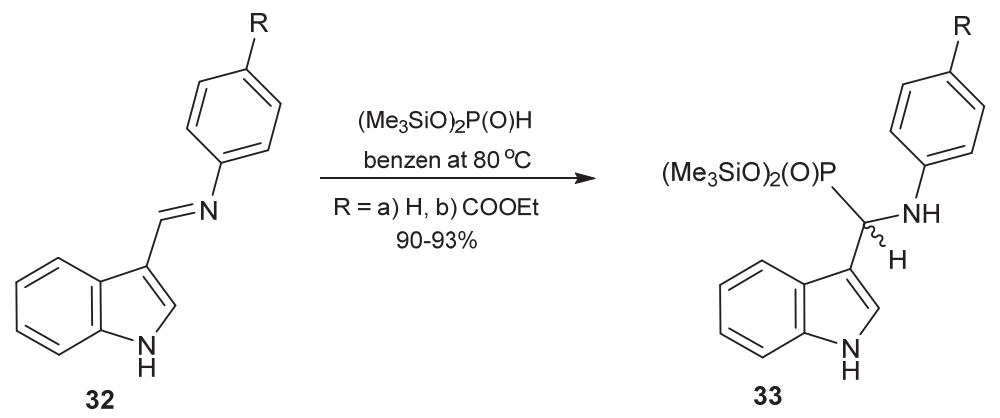

33

\section{Scheme 12}

Reaction of the Schiff bases 34 with diethyl hydrogen phosphite via Pudovik reaction in refluxing toluene in the absence of catalyst afforded the corresponding $\alpha$-aminophosphonates 35 (Scheme 13). ${ }^{34}$ 


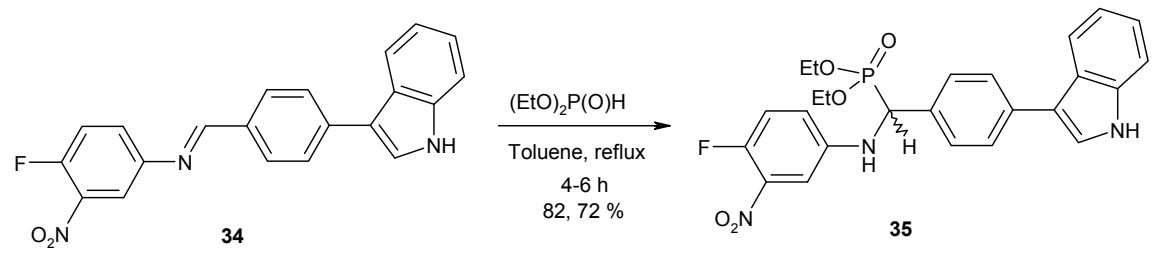

\section{Scheme 13}

\subsection{Five-membered heterocycles with two heteroatoms}

The aldimines 36 were reacted directly with trimethyl phosphite in the presence of bromotrimethylsilane to form the $\alpha$-(2-thiazolyl)- $\alpha$-aminophosphonic silylated esters $\mathbf{3 7}$, as intermediates, which were then deprotected giving the $\alpha$-(2-thiazolyl)- $\alpha$-aminophosphonic acids 38 (Scheme 14). ${ }^{35}$

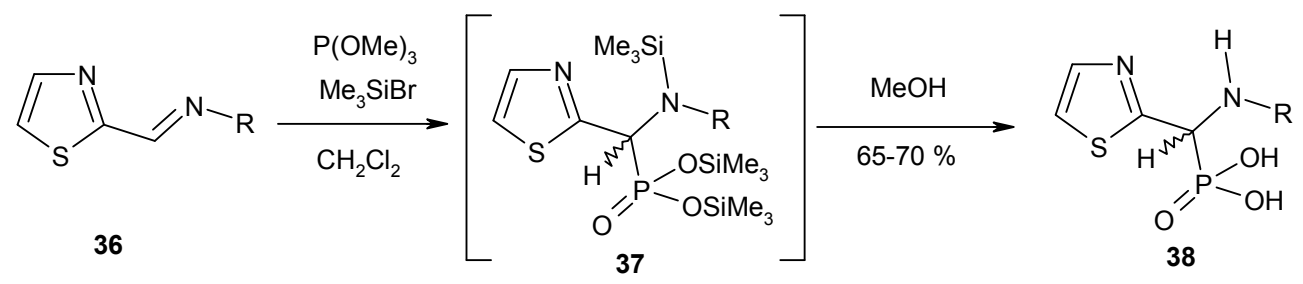

$\mathrm{R}=n-\mathrm{Bu}, \mathrm{Ph}, \mathrm{CH}_{2} \mathrm{Ph}$

\section{Scheme 14}

Similarly, the reaction of imines 39 with tris(trimethylsilyl) phosphite (generated in situ from triethyl or trimethyl phosphite and bromotrimethylsilane) to give the silylated intermediates $\mathbf{4 0}$ which were then treated with methanol, producing the desired desilyated $\alpha$-(imidazol-2-yl)- $\alpha$ aminophosphonic acids 41 (Scheme 15). ${ }^{36,37}$

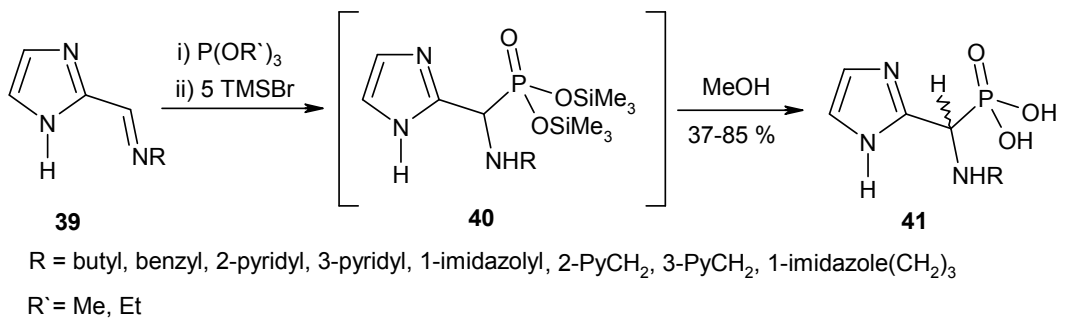

\section{Scheme 15}

The diethyl 1,3-benzodioxylphosphonate esters $\mathbf{4 3}$ were prepared by heating equimolar mixtures of diethyl phosphite and the corresponding Schiff base $\mathbf{4 2}$ in the absence of solvent at temperatures between 90 and $100{ }^{\circ} \mathrm{C}($ Scheme 16$) .{ }^{25}$ 


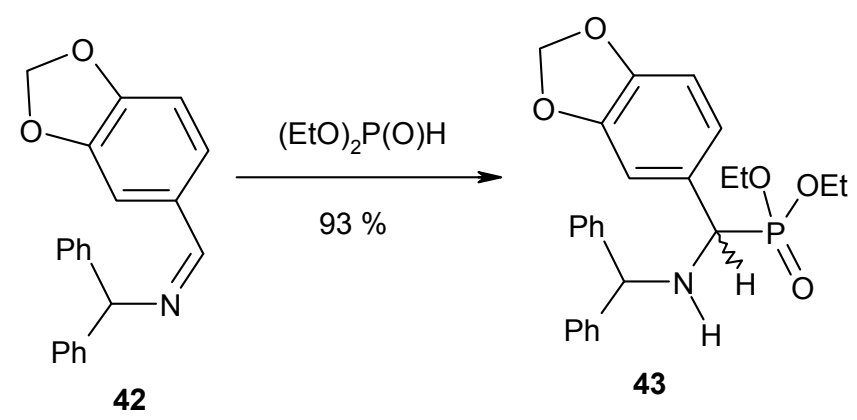

\section{Scheme 16}

\subsection{Six-membered heterocycles with one heteroatom}

Addition of diethyl phosphite to the Schiff base $\mathbf{4 4}$ at room temperature without a solvent in the presence of catalytic amounts of sodium ethoxide afforded diethyl [( $N$-benzylamino)(2pyridinyl)methyl]phosphonate (45) which underwent acidic hydrolysis to give the corresponding $\alpha$-aminophosphonic acid 46 (Scheme 17). ${ }^{38}$

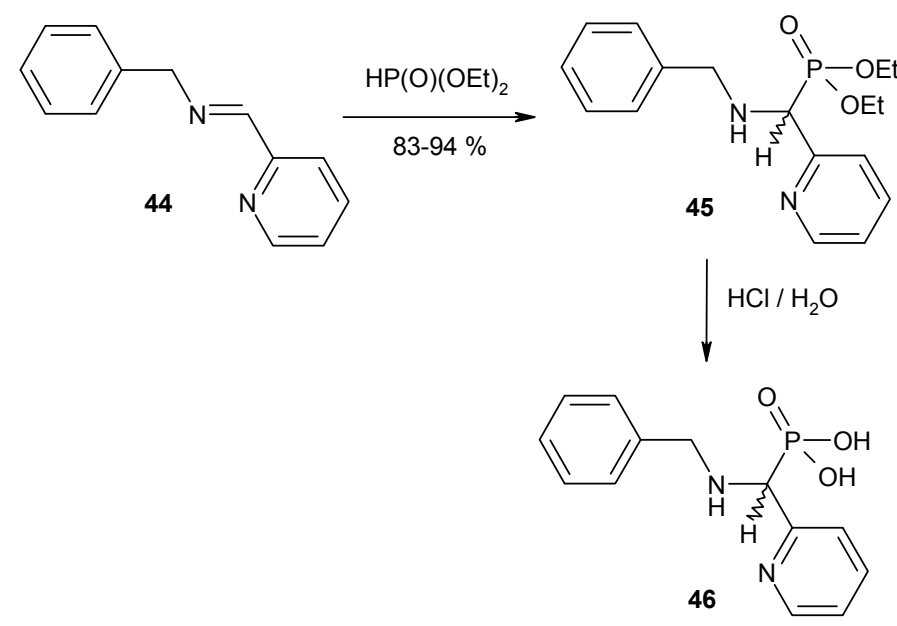

\section{Scheme 17}

1-( $N$-Benzyl)-2-formyl-5-benzyloxy-pyridone 47 reacted with primary amines to obtain the corresponding imines 48 . The imines then were treated with a mixture of trimethyl phosphite and bromotrimethylsilane, which caused in situ formation of tris(trimethylsilyl) phosphite, which instantly reacted with the imines, giving silylated phosphonate intermediates. Treatment of the intermediates with methanol caused removal of the silylated groups and the formation of the final $\alpha$-(pyridinyl)- $\alpha$-aminophosphonic acids 49 (Scheme 18). ${ }^{39}$ 


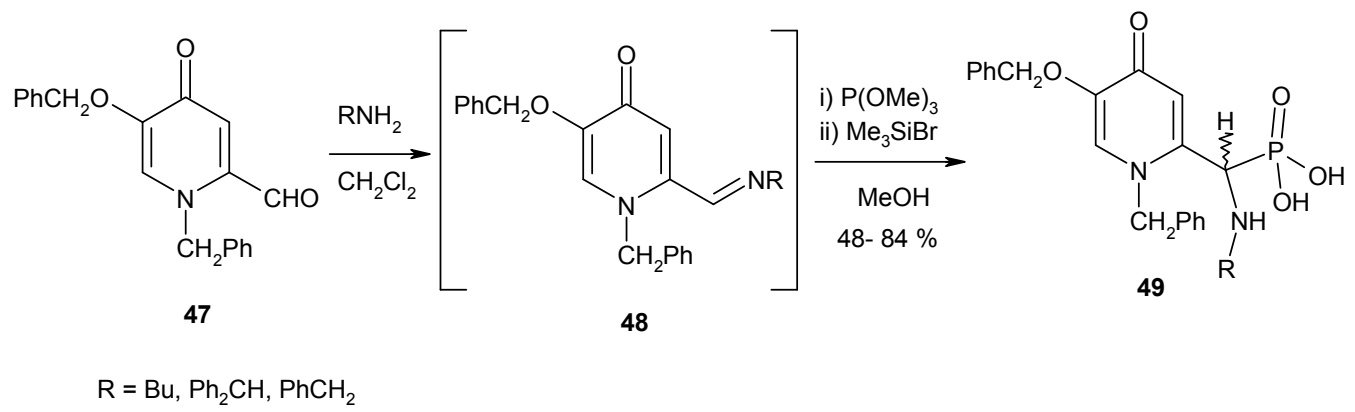

\section{Scheme 18}

A short and efficient synthesis of new 4-amino(piperidine/tetrahydropyran/ tetrahydrothiopyran)-4-phosphonic acids $\mathbf{5 2}$ in good yields was described via addition of triethyl phosphite in acidic medium to ketone imines $\mathbf{5 0}$ via $\alpha$-aminophosphonates $\mathbf{5 1}$ (Scheme 19). ${ }^{27}$

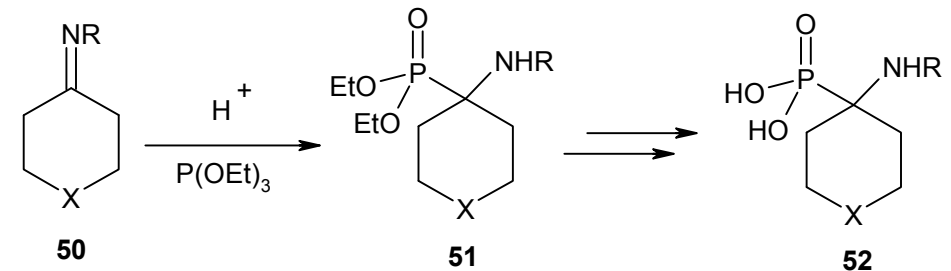

$\mathrm{X}=\mathrm{O}, \mathrm{S}, \mathrm{NR}$

\section{Scheme 19}

Some piperidine-incorporated $\alpha$-aminophosphonates 54 were prepared in excellent yields by reacting imines $\mathbf{5 3}$ with triethyl phosphite in the presence of dilute $\mathrm{HCl}$ under ultrasound irradiation (Scheme 20). ${ }^{40}$

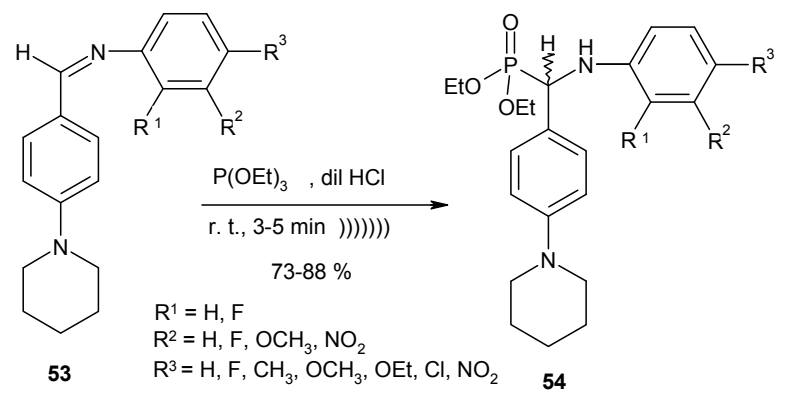

\section{Scheme 20}

$\alpha$-(3-Quinolinyl)- $\alpha$-aminophosphonates 56 were prepared in quantitative yields by reacting imines 55 with triethyl phosphite in the presence of tetramethylsilyl chloride (TMSCl) at room 
temperature. The yields of the $\alpha$-aminophosphonates using this process are in the range of $95-$ $98 \%$ (Scheme 21). ${ }^{41,42}$

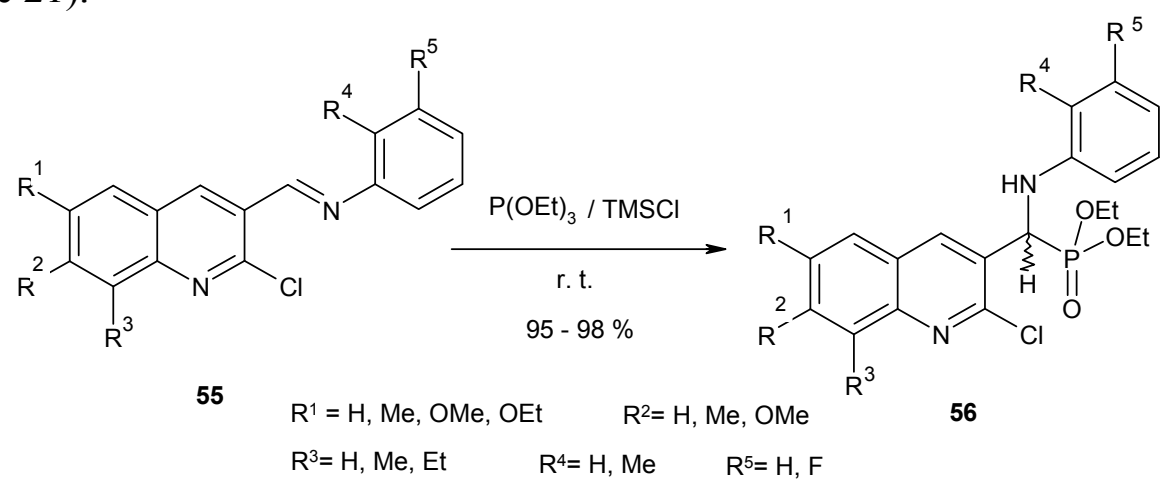

\section{Scheme 21}

Nucleophilic addition of the silylated phosphorus ester to imines $\mathbf{5 7}$ proceeded easily at room temperature for 12 hours. The formed silylated phosphonic intermediates $\mathbf{5 8}$ were treated with methanol as a desilylating agent to produce the desired $\alpha$-(quinolin-2, 3- and 4-yl)- $\alpha$ (amino)methylphosphonic acids 59 in good yields (Scheme 22). ${ }^{43}$

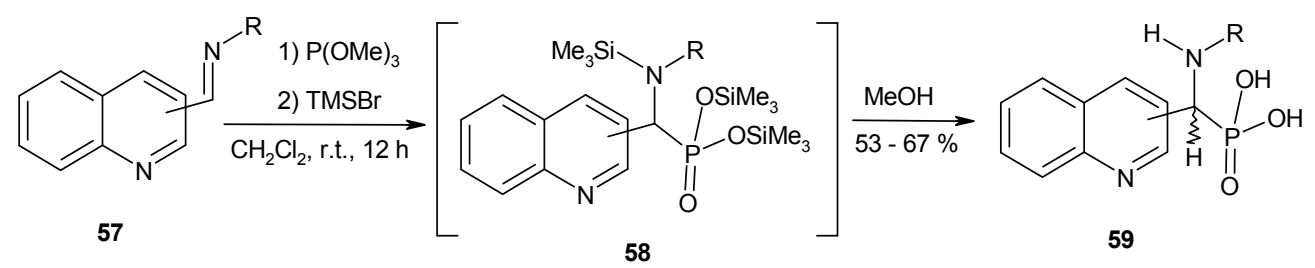

\section{Scheme 22}

The synthesized imines $\mathbf{6 0}$ were treated with triethyl phosphite in the presence of $\mathrm{TMSCl}$ at room temperature to afford the corresponding diethyl $\alpha$-(tetrazoloquinolin-3-yl)- $\alpha$-aminophosphonate 61 (Scheme 23). ${ }^{44}$<smiles>[R]c1cc2cc(/C=N/c3ccc(F)cc3)c3nnnn3c2c([R])c1[R]</smiles>

60

61

$\mathrm{R}^{1}=\mathrm{H}, \mathrm{Me}$, OMe, OEt

$\mathrm{R}^{2}=\mathrm{H}, \mathrm{Me}, \mathrm{OMe}$

$\mathrm{R}^{3}=\mathrm{H}, \mathrm{Me}, \mathrm{Et}, \mathrm{OMe}$

\section{Scheme 23}


The Schiff bases 62 reacted with dialkyl phosphite or trialkyl phosphite in presence or absence of solvent to give $\alpha$-(chromon-3-yl)- $\alpha$-aminophosphonates $\mathbf{6 3}$ (Scheme 24). ${ }^{18,45,46}$

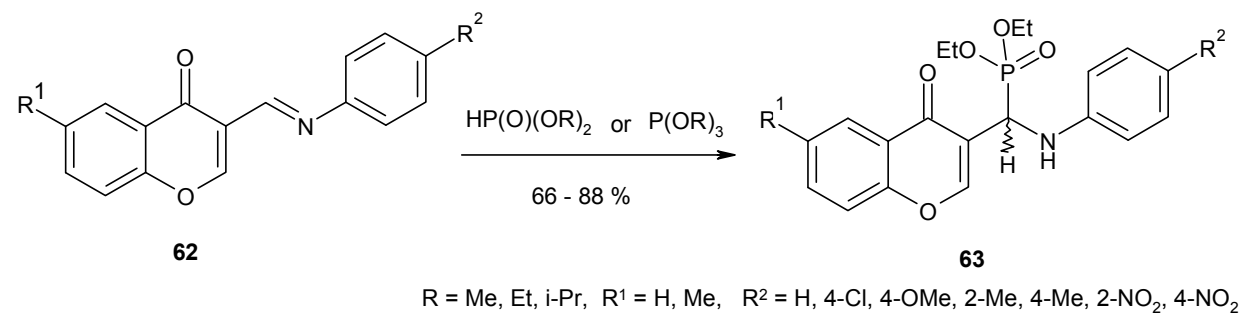

\section{Scheme 24}

Fusing of the bis-phosphonic hydrazone 64 with diethyl phosphite at $80-100{ }^{\circ} \mathrm{C}$ in the presence of catalytic amounts of triethylamine produced $\mathrm{N}^{1}, \mathrm{~N}^{5}$-bis $[N$-methyl(diethoxyphosphonyl)-1-[(4-oxo-4H-chromen-3-yl)]phosphonic dihydrazide (65) as the sole product (Scheme 25). ${ }^{47}$

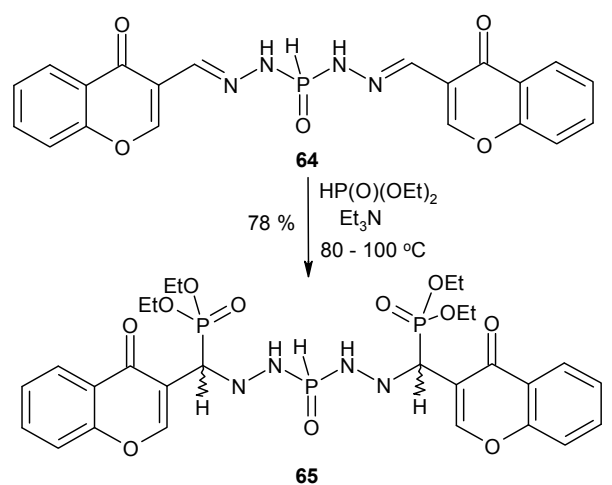

\section{Scheme 25}

Similarly, the addition of diethyl phosphite to compounds 66 was carried out in dry benzene containing few drops of triethylamine as catalyst to yield the corresponding bis-( $\alpha$-aminophosphonate) derivatives 67 (Scheme 26). ${ }^{47}$

Also, addition of diethyl phosphite to azomethine bonds of interesting compounds 68 and 69 on fusion at $80-100{ }^{\circ} \mathrm{C}$ in the presence of a catalytic amount of triethylamine yielded one diastereomeric form of tetraethyl 5,5'-(1,4-phenylene)bis-[[[(3-oxo-3-phenyl-2,3-dihydro-4H1,2,4,3-triazaphosphol-4-yl)amino](4-oxo-4H-chromen-3-yl)methyl]phosphonate] (70) and two diastereomeric forms of tetraethyl 5,5'-(1,4-phenylene)bis-[[[(3-oxo-3-phenyl-2,3-dihydro-4H1,2,4,3-triazaphosphol-4-yl)methylphosphoryl]amino](6-methyl-4-oxo-4H-chromen-3-yl)methyl] phosphonate] (71), respectively (Scheme 27). ${ }^{17}$ 


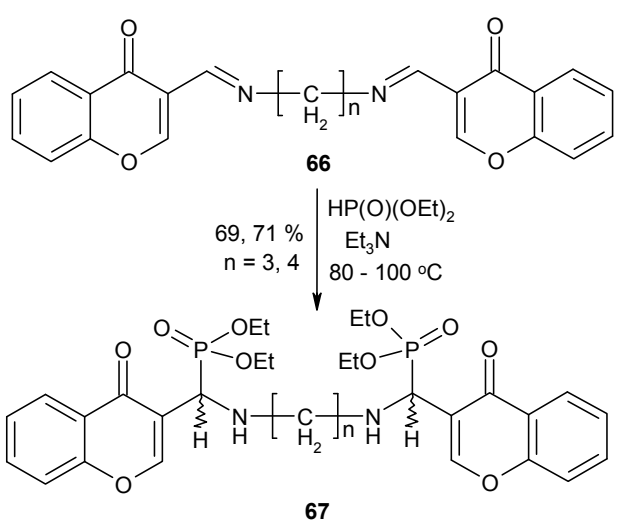

\section{Scheme 26}

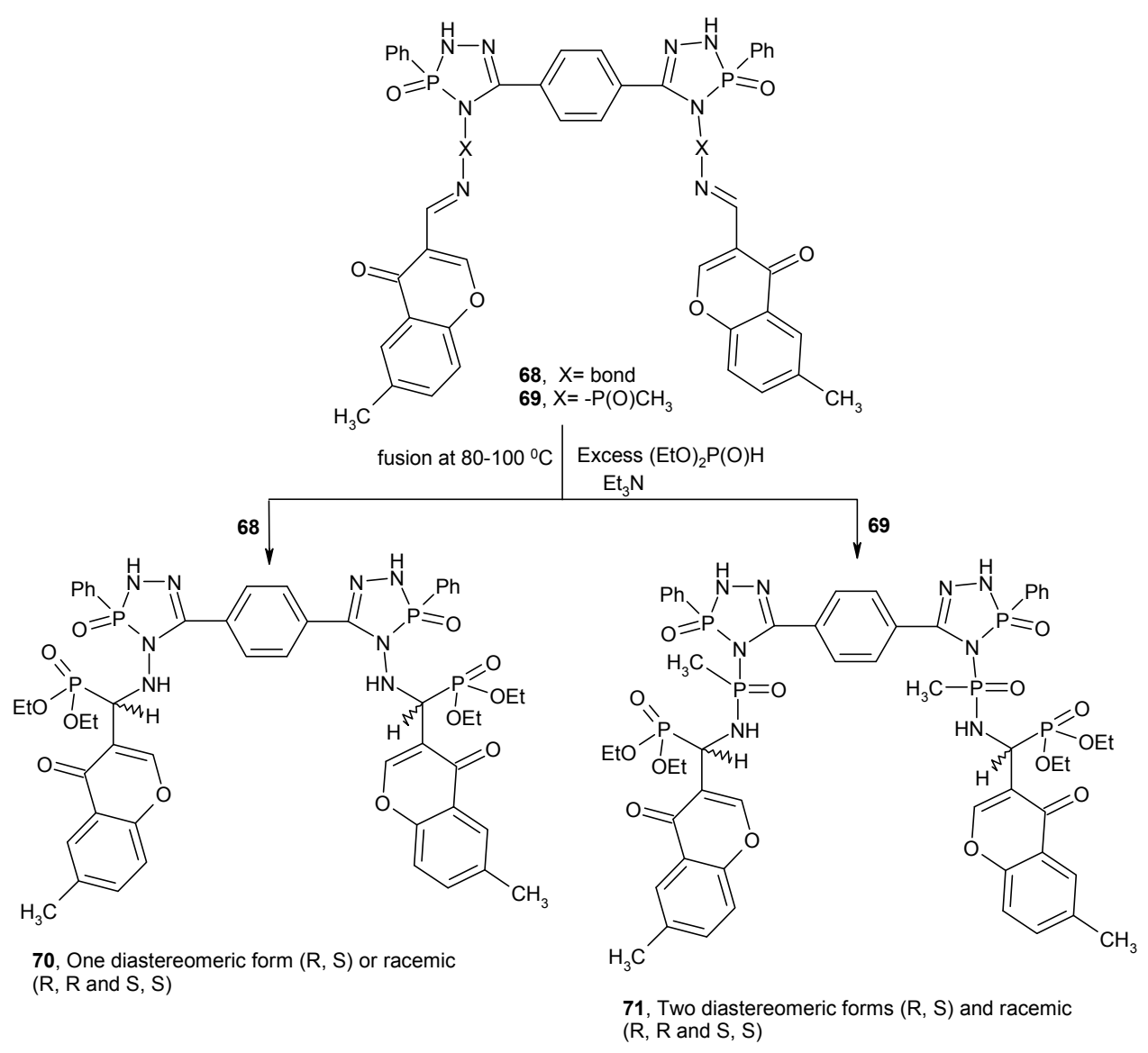

Scheme 27

\section{Synthesis by Kabachnik-Fields Reaction}

\subsection{Five-membered heterocycles with one heteroatom}

Three component one-pot reaction of heterocyclic aldehydes such as furan-2-carbaldehyde, 
thiophene-2-carbaldehyde and pyrrole-2-carbaldehyde with different amines such as butylamine, cyclohexylamine, aniline, benzylamine, 4-chloroaniline, 4-methoxyaniline, 4-fluoroaniline, 4-methoxyaniline, HMDS (1,1,1,3,3,3-hexamethyldisilazane), aminoalkylphosphonic acid and heteroaryl amines with dialkyl or trialkyl phosphites gave the corresponding $\alpha$-heterocyclic- $\alpha$ aminophosphonates $\mathbf{7 2}$ in good to excellent yields under different reaction conditions (Scheme 28) (Table 1). ${ }^{10,20,48-68}$

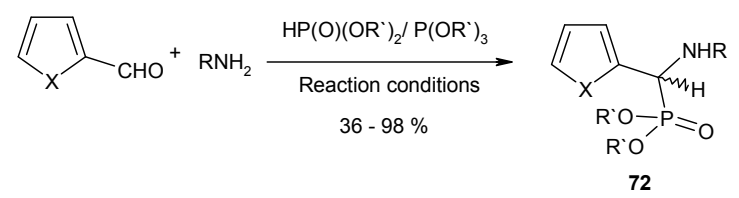

\section{Scheme 28}

Table 1. Conditions of reactions of furan-, thiophene-, or pyrrole-2-carbaldehyde and different amines with dialkyl or trialkyl phosphites to give the corresponding $\alpha$-heterocyclic- $\alpha$ aminophosphonates $\mathbf{7 2}$

\begin{tabular}{|c|c|c|c|c|c|}
\hline $\begin{array}{l}\text { Entry } \\
\text { No. }\end{array}$ & $\mathrm{X}$ & $\mathrm{R}$ in Scheme 28 & $\mathrm{R}^{\prime}$ & Reaction conditions & Ref. \\
\hline 1 & $\mathrm{O}, \mathrm{S}, \mathrm{NH}$ & $\mathrm{Bu}, \mathrm{PhCH}_{2}$ & Et, $\mathrm{Ph}, \mathrm{PhCH}_{2}$ & toluene, reflux & 10 \\
\hline 2 & $\mathrm{O}$ & $4-\mathrm{MeOC}_{6} \mathrm{H}_{4}$ & Et & $\mathrm{NH}_{4} \mathrm{VO}_{3} / \mathrm{RT} /$ stirring & 49 \\
\hline 3 & $\mathrm{O}, \mathrm{S}$ & $\mathrm{Ph}$ & Et & {$[$ bnmim $]\left[\mathrm{HSO}_{4}\right] / \mathrm{RT} /$ stirring } & 50 \\
\hline 4 & $\mathrm{O}, \mathrm{S}$ & $\mathrm{Ph}$ & Et & 1-hexanesulfonic acid sodium salt/ultrasound & 51 \\
\hline 5 & S & $\mathrm{Ph}$ & $\mathrm{Me}$ & $\mathrm{AlCl}_{3} / \mathrm{CH}_{3} \mathrm{CN} / \mathrm{RT}$ & 52 \\
\hline 6 & $\mathrm{O}, \mathrm{S}$ & $\mathrm{Ph}, 4-\mathrm{FC}_{6} \mathrm{H}_{4}$ & Et & {$[\mathrm{bmim}] \mathrm{BF}_{4}$ or $[\mathrm{bmim}] \mathrm{PF}_{6}$} & 54 \\
\hline 7 & $\mathrm{O}$ & $\mathrm{Ph}, 4-\mathrm{MeOC}_{6} \mathrm{H}_{4}$ & Et & $10 \mathrm{~mol} \%, \mathrm{GaI}_{3} / \mathrm{CH}_{2} \mathrm{Cl}_{2} / \mathrm{RT}$ & 55 \\
\hline 8 & $\mathrm{O}$ & $\mathrm{PhCH}_{2}$ & $\mathrm{Et}$ & $\mathrm{LiClO}_{4}, 20 \mathrm{~mol} \%, 60{ }^{\circ} \mathrm{C}, 8 \mathrm{~h}$ & 56 \\
\hline 9 & $\mathrm{O}, \mathrm{S}$ & $4-\mathrm{MeOC}_{6} \mathrm{H}_{4}$ & $2-\mathrm{MeOC}_{6} \mathrm{H}_{4}$ & $\mathrm{Zn}\left(\mathrm{NTf}_{2}\right)_{2} / 10 \mathrm{~mol} \%, \mathrm{CH}_{2} \mathrm{Cl}_{2},-50^{\circ} \mathrm{C}$ & 57 \\
\hline 10 & $\mathrm{O}, \mathrm{S}$ & {$\left[\left(\mathrm{Me}_{3} \mathrm{Si}\right)_{2} \mathrm{NH}\right]$} & Et & $\mathrm{I}_{2}(10 \mathrm{~mol} \%)$, solvent free & 58 \\
\hline 11 & $\mathrm{~S}$ & $\left(\mathrm{CH}_{2}\right)_{\mathrm{n}} \mathrm{COOH}$ & Et & $\mathrm{MeOH}, \mathrm{Et}_{3} \mathrm{~N}$ & 61 \\
\hline 12 & $\mathrm{O}$ & $4-\mathrm{MeOC}_{6} \mathrm{H}_{4}$ & $\mathrm{Me}$ & Yttria-zirconia Lewis acid/ aq. $\mathrm{CH}_{3} \mathrm{CN} / 60^{\circ} \mathrm{C}$ & 48 \\
\hline 13 & $\mathrm{O}, \mathrm{S}$ & $\mathrm{Ph}$ & Et & $\begin{array}{c}{[\mathrm{Cu}(3,4 \text {-tmtppa })]\left(\mathrm{MeSO}_{4}\right) /(0.16 \% \mathrm{~mol})} \\
/ \mathrm{H}_{2} \mathrm{O} / 80{ }^{\circ} \mathrm{C}\end{array}$ & 53 \\
\hline 14 & $\mathrm{O}, \mathrm{S}, \mathrm{NH}$ & {$\left[\left(\mathrm{Me}_{3} \mathrm{Si}\right)_{2} \mathrm{NH}\right]$} & Et & $\mathrm{Al}(\mathrm{OTf})_{3}(10 \mathrm{~mol} \%) /$ solvent free/ $80^{\circ} \mathrm{C}$ & 59 \\
\hline 15 & $\mathrm{O}, \mathrm{NH}$ & $\mathrm{Ph}, 4-\mathrm{MeOC}_{6} \mathrm{H}_{4}$ & Et, $\mathrm{PhCH}_{2}$ & $\mathrm{H}-\beta$-zeolite, heat, $\mathrm{MeCN}$ & 60 \\
\hline 16 & $\mathrm{~S}$ & $\mathrm{PhCH}_{2}$ & Et & toluene, reflux, $3 \mathrm{~h}$ & 20 \\
\hline 17 & $\mathrm{~S}$ & $\mathrm{Ph}$ & Et & $\mathrm{TiO}_{2}(20 \mathrm{~mol} \%)$, no solvent, $50^{\circ} \mathrm{C}$ & 62 \\
\hline 18 & $\mathrm{O}$ & $\mathrm{Ph}, 3$-pyridinyl & Et & Metal oxide, ultrasound & 63 \\
\hline 19 & $\mathrm{O}$ & $\mathrm{Ph}$ & $\mathrm{Me}$ & $\begin{array}{c}\text { Homogeneous sulphamic acid }(0.7 \mathrm{~mol} \%) \text {, } \\
\text { neat/RT }\end{array}$ & 64 \\
\hline
\end{tabular}


Table 1. Continued

\begin{tabular}{|c|c|c|c|c|c|}
\hline $\begin{array}{l}\text { Entry } \\
\text { No. }\end{array}$ & $\mathrm{X}$ & $\mathrm{R}$ in Scheme 28 & $\mathrm{R}^{\prime}$ & Reaction conditions & Ref. \\
\hline 20 & $\mathrm{O}$ & $\begin{array}{l}\text { 4-phenoxyquinazolin- } \\
\text { 2-yl }\end{array}$ & Et & {$[\mathrm{BMIM}] \mathrm{Cl} / \mathrm{MW}$} & 65 \\
\hline 21 & $\mathrm{O}, \mathrm{S}$ & $\mathrm{Ph}$ & $\mathrm{Me}, \mathrm{Et}$ & $\mathrm{Mg}\left(\mathrm{ClO}_{4}\right)_{2}(5 \mathrm{~mol} \%)$ & 66 \\
\hline 22 & $\mathrm{~S}$ & 1-(furan-2-yl)methyl & $\mathrm{Me}$ & $\begin{array}{l}\text { TMG (tetramethyl guanidine), toluene, } \\
\qquad 50-60^{\circ} \mathrm{C}, 5-6 \mathrm{~h}\end{array}$ & 67 \\
\hline 23 & $\mathrm{O}, \mathrm{S}$ & $\begin{array}{c}\text { Dibenzo }\left[d_{f} f\right][1,3,2] \\
\text { diazaphosphepin-6-yl } \\
\text { 6-oxide- }\end{array}$ & $\mathrm{Me}, \mathrm{Et}$ & TMG, toluene, $60-70^{\circ} \mathrm{C}, 4 \mathrm{~h}$ & 68 \\
\hline
\end{tabular}

Refluxing thiophene-2-carbaldehyde with a mixture of urea and diethyl phosphite in dry toluene afforded the ureidophosphonate $\mathbf{7 3}$ as a major product (Scheme 29). ${ }^{69}$

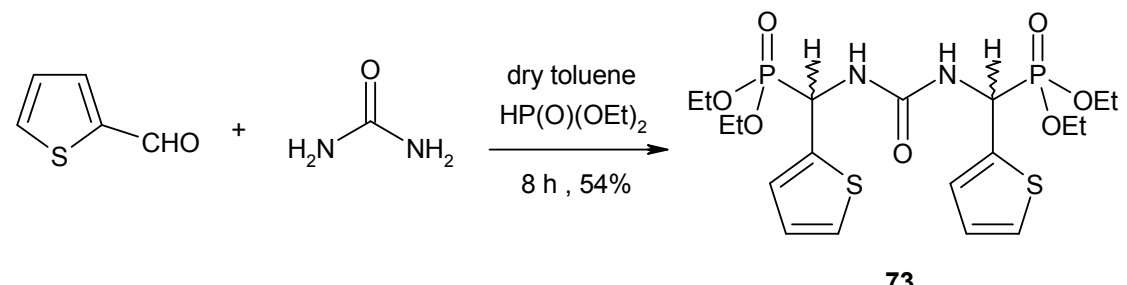

\section{Scheme 29}

Magnetic iron oxide nanoparticles coated with structurally variable $\alpha$-heterocyclic- $\alpha$ aminophosphonates 75 have been obtained by one-pot three-component reaction of 2-aminopyridine iron oxide nanoparticle $\mathbf{7 4}$, heterocyclic aldehydes and diethyl phosphite (Scheme 30$).^{70}$

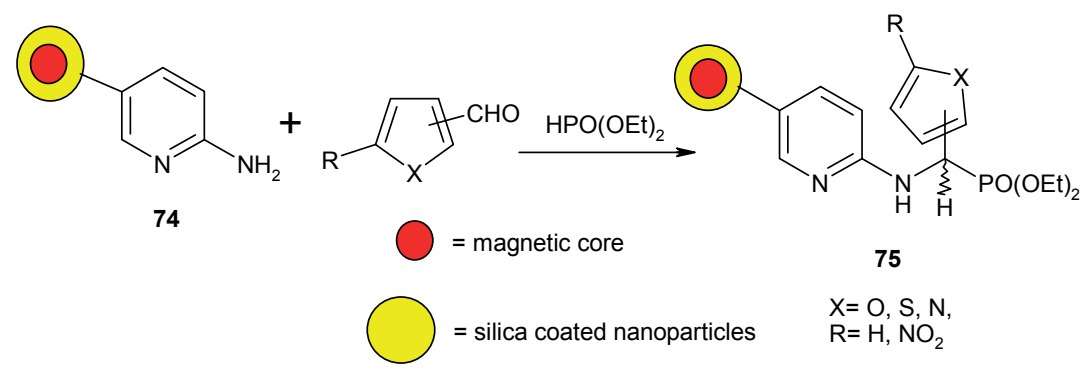

\section{Scheme 30}

$\alpha$-(Indol-2-yl or 3-yl)- $\alpha$-aminopyridinylphosphonates $\mathbf{7 6}$ were obtained from the corresponding indole aldehydes, 2-aminopyridine and diethyl phosphite without any solvent (Scheme 31). ${ }^{71,72}$ 


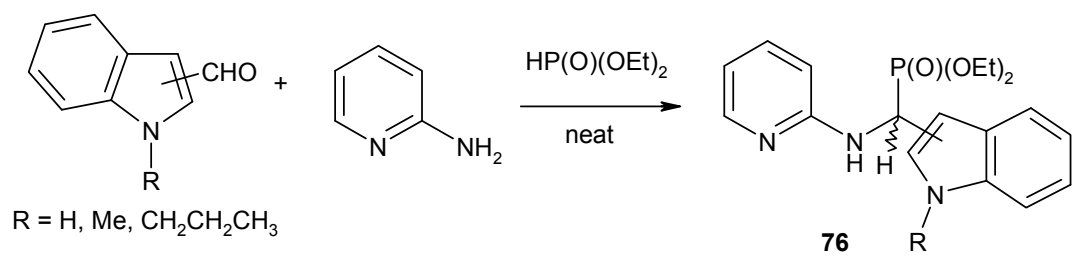

\section{Scheme 31}

Diethyl $\alpha$-(indol-3-yl)- $\alpha$-aminophosphonates 77 were synthesized through the reaction of 3formylindole, diethyl phosphite and heterocyclic amines and/or ammonium carbonate or ammonium acetate under various reaction conditions (Scheme 32) (Table 2). ${ }^{58-60,68,73,74}$
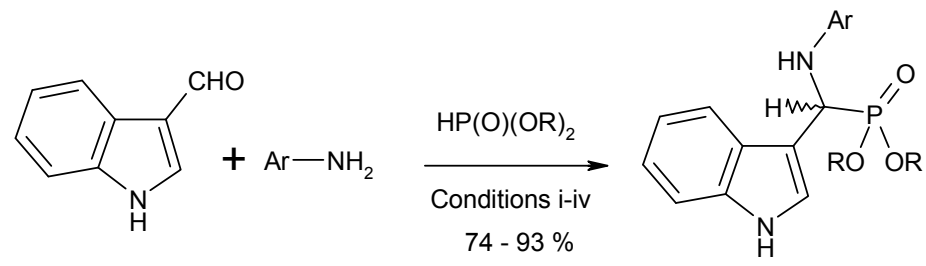

77

\section{Scheme 32}

Table 2. Conditions reaction of 3-formylindole, diethyl phosphite and amines to give diethyl indolyl- $\alpha$-aminophosphonates 77

\begin{tabular}{|c|c|c|c|c|}
\hline Entry No. & Conditions & Amine & $\mathrm{R}$ & Ref. \\
\hline 1 & $\mathrm{I}_{2}(10 \mathrm{~mol} \%) /$ solvent-free & $\left(\mathrm{Me}_{3} \mathrm{Si}\right)_{2} \mathrm{NH}$ & Et & 58 \\
\hline 2 & $\begin{array}{c}\mathrm{Al}(\mathrm{OTf})_{3}(10 \mathrm{~mol} \%), \text { solvent- } \\
\text { free } / 80^{\circ} \mathrm{C}\end{array}$ & $\left(\mathrm{Me}_{3} \mathrm{Si}\right)_{2} \mathrm{NH}$ & Et & 59 \\
\hline 3 & $\begin{array}{c}\mathrm{Al}(\mathrm{OTf})_{3}(10 \mathrm{~mol} \%), \text { solvent- } \\
\text { free } / 100^{\circ} \mathrm{C}\end{array}$ & $\left(\mathrm{NH}_{4}\right)_{2} \mathrm{CO}_{3}$ or $\mathrm{NH}_{4} \mathrm{OAc}$ & Et & 73 \\
\hline 4 & $\beta$-zeolite & $\mathrm{PhNH}_{2}, 4-\mathrm{MeOC}_{6} \mathrm{H}_{4} \mathrm{NH}_{2}$ & Et & 60 \\
\hline 5 & $\mathrm{PEG} / \mathrm{H}_{2} \mathrm{O} / \mathrm{RT}$ & & Et & 74 \\
\hline 6 & $\begin{array}{c}\text { toluene / TMG / } \\
60-70{ }^{\circ} \mathrm{C} / 4 \mathrm{~h}\end{array}$ & & $\mathrm{Me}, \mathrm{Et}$ & 68 \\
\hline
\end{tabular}

$\alpha$-(Indol-3-yl)- $\alpha$-aminophosphonate 79 was synthesized by the reaction of an aromatic amine, 3-(4-formylphenyl)indole (78) and diethyl phosphite under MW conditions in the presence of Amberlyst-15 (Scheme 33). ${ }^{75}$ 


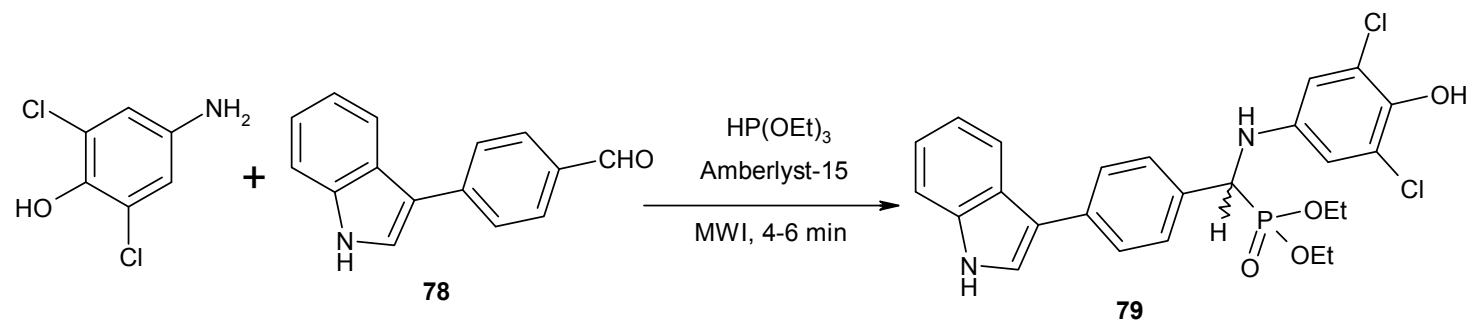

\section{Scheme 33}

Various substituted anilines carrying either electron donating or electron withdrawing substituents, and also benzylamine, reacted with 9-ethyl-6-bromo-3-formylcarbazole (80) and diethyl phosphite in PEG-mediated reactions to give the desired $\alpha$-(carbazol-3-yl)- $\alpha$-aminophosphonates $\mathbf{8 1}$ in good yields. In this reaction PEG-400 not only acts as the solvent but also accelerates the imine formation and the nucleophilic addition of phosphite to the imine by increasing its electrophilicity through hydrogen bonding by its hydroxyl group with the imine nitrogen (Scheme 34). ${ }^{76}$

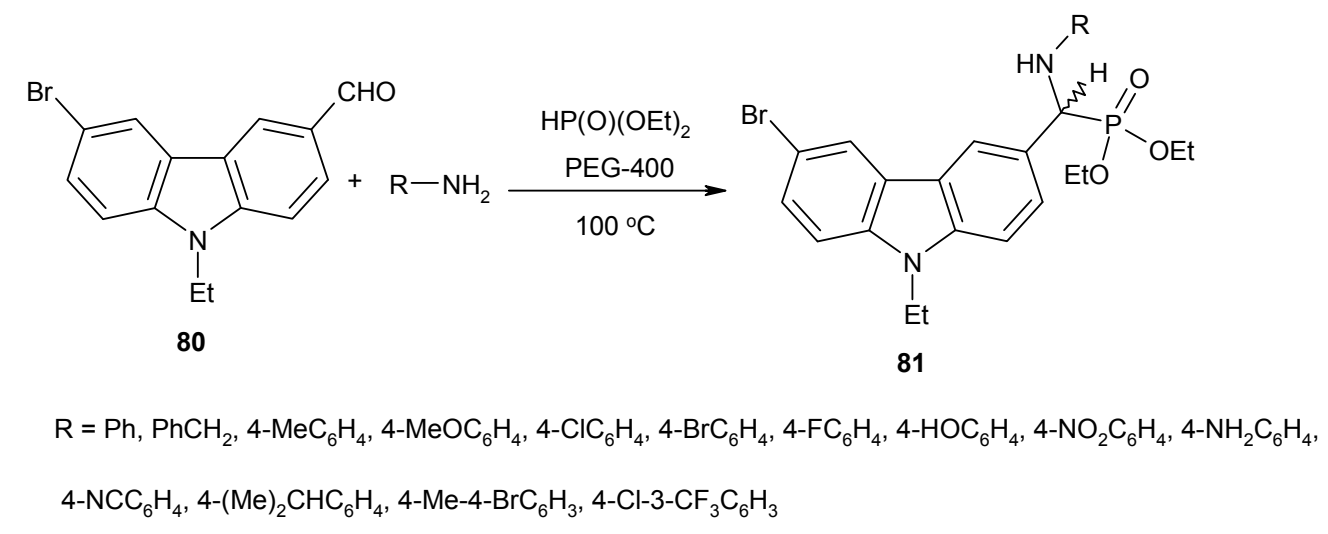

\section{Scheme 34}

\subsection{Five-membered heterocycles with two heteroatoms}

In a one-pot synthesis, 3-formylpyrazole (82) reacted with benzyl carbamate and triphenyl phosphite in acetic acid to give $\alpha$-(pyrazol-3-yl)- $\alpha$-aminophosphonate $\mathbf{8 3}$ in moderate yield (Scheme 35). ${ }^{20}$ 


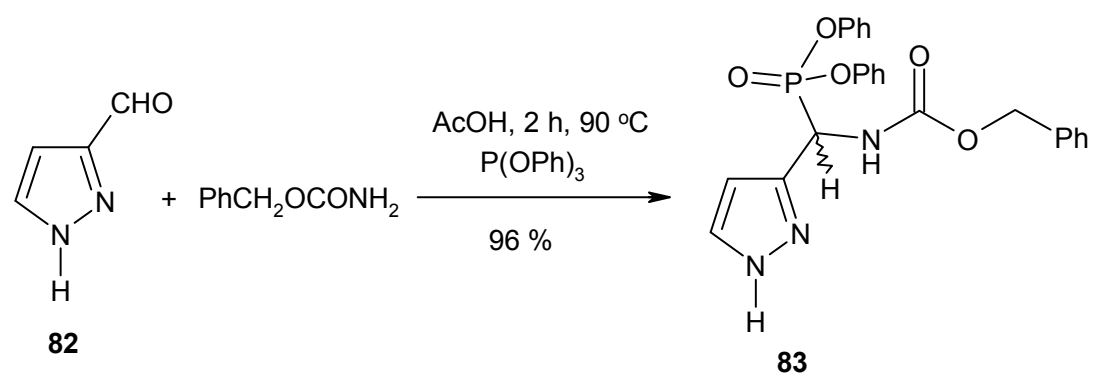

\section{Scheme 35}

1-Phenyl-3-aryl/heteroaryl-1H-pyrazol-4-carboxaldehyde (84) are reported to react with arylamines and dialkyl/diphenyl phosphites under Kabachnik-Fields reaction conditions in the presence or absence of catalysts to give the corresponding $\alpha$-(pyrazol-4-yl)- $\alpha$-aminophosphonates 85 (Scheme 36) (Table 3). ${ }^{77-80}$
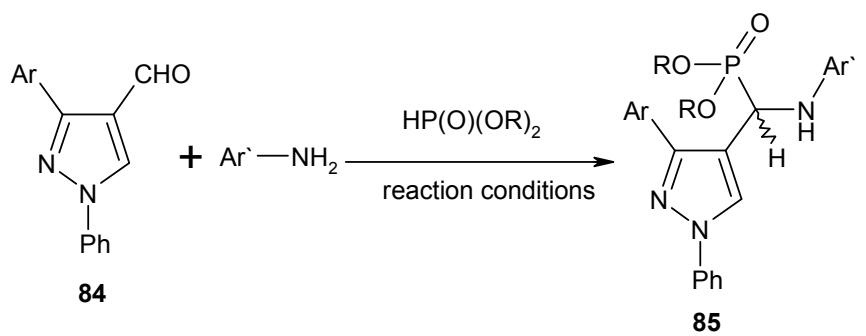

\section{Scheme 36}

Table 3. Conditions of reactions of 1-phenyl-3-aryl/heteroaryl-1H-pyrazol-4-carboxaldehyde (84) with arylamines and dialkyl/diphenyl phosphites to give pyrazolyl- $\alpha$-aminophosphonates $\mathbf{8 5}$

\begin{tabular}{|c|c|c|c|c|c|}
\hline $\begin{array}{c}\text { Entry } \\
\text { No. }\end{array}$ & $\mathrm{Ar}$ & $\mathrm{Ar}^{\prime}$ & $\mathrm{R}$ & Conditions & Ref. \\
\hline 1 & $\mathrm{Ph}$ & $\mathrm{Ph}$ & Et & $\mathrm{BF}_{3} \cdot \mathrm{Et}_{2} \mathrm{O} / 70-80^{\circ} \mathrm{C}$ & 77 \\
\hline 2 & $4-\mathrm{ClC}_{6} \mathrm{H}_{4}$ & $\begin{array}{c}\mathrm{Ph}, 4-\mathrm{MeC}_{6} \mathrm{H}_{4}, 4-\mathrm{ClC}_{6} \mathrm{H}_{4}, \\
\text { 4- } \mathrm{BrC}_{6} \mathrm{H}_{4}, 4-\mathrm{IC}_{6} \mathrm{H}_{4}, 4-\mathrm{FC}_{6} \mathrm{H}_{4}, \\
\text { 3-Cl-4- } \mathrm{FC}_{6} \mathrm{H}_{3}, 3,4-\mathrm{Me}_{2} \mathrm{C}_{6} \mathrm{H}_{3}, \\
\text { 4- } \mathrm{MeOC}_{6} \mathrm{H}_{4}, 4-\mathrm{EtOC}_{6} \mathrm{H}_{4}\end{array}$ & $\mathrm{Et}$ & $\mathrm{MW}(200 \mathrm{~W}), 80^{\circ} \mathrm{C}, 3 \mathrm{~min}$ & 80 \\
\hline 3 & 2-pyridyl & $\begin{array}{c}\mathrm{Ph}, 4-\mathrm{MeC}_{6} \mathrm{H}_{4}, 4-\mathrm{ClC}_{6} \mathrm{H}_{4} \\
\text { 4- } \mathrm{HOC}_{6} \mathrm{H}_{4}, 4-\mathrm{MeOC}_{6} \mathrm{H}_{4} \\
\text { 4- } \mathrm{Me}_{2} \mathrm{NC}_{6} \mathrm{H}_{4}\end{array}$ & $\mathrm{Ph}$ & $\mathrm{LiClO}_{4}, \mathrm{DCM}, \mathrm{RT}, 24-30 \mathrm{~h}$ & 79 \\
\hline 4 & $\mathrm{Ph}, 4-\mathrm{MeOC}_{6} \mathrm{H}_{4}$ & $\begin{array}{c}\mathrm{Ph}, 4-\mathrm{MeC}_{6} \mathrm{H}_{4}, 2-\mathrm{MeC}_{6} \mathrm{H}_{4}, \\
4-\mathrm{MeOC}_{6} \mathrm{H}_{4}\end{array}$ & $\mathrm{Me}, \mathrm{Et}$ & Toluene/ $110^{\circ} \mathrm{C}$ & 78 \\
\hline
\end{tabular}


One-pot three-component reaction of bis-(4-formyl-3-phenyl-1H-pyrazol-1-yl)phosphine oxide (86), aniline and diethyl phosphite in the presence of $\mathrm{BF}_{3} . \mathrm{Et}_{2} \mathrm{O}$ at $80{ }^{\circ} \mathrm{C}$ under KabachnikFields reaction conditions produced an interesting type of bis $\alpha$-aminophosphonate) 87 (Scheme $37)^{81}$

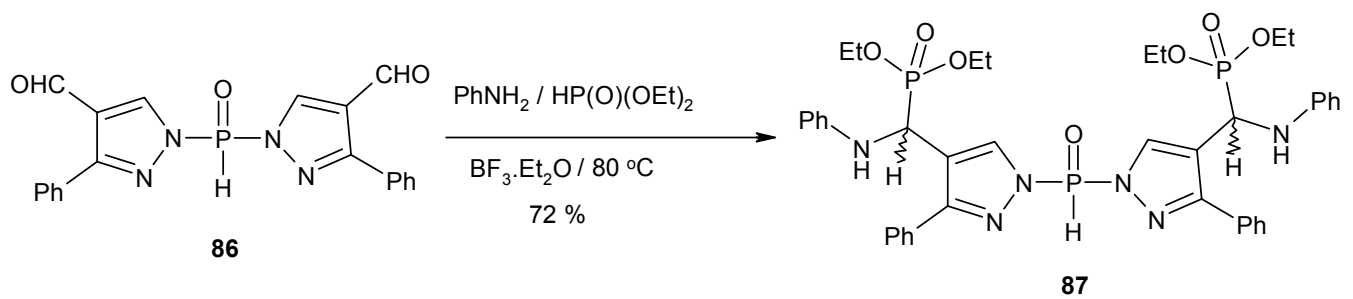

\section{Scheme 37}

Reaction of 6-amino- $6 \lambda^{5}$-dibenzo[ $[d, f][1,3,2]$ dioxaphosphepin-6-oxide (88) with 2-formylimidazole (89) and dialkyl phosphites in dry toluene in the presence of tetramethylguanidine (TMG) as a catalyst at $60-70{ }^{\circ} \mathrm{C}$ for 4 hours afforded dimethyl/diethyl(6-oxo- $6 \lambda^{5}$-dibenzo $[d, f]$ $[1,3,2]$ dioxaphosphepin-6-ylamino)-(1H-2-imidazolyl)methylphosphonates (90) in good yields (Scheme 38). ${ }^{68}$
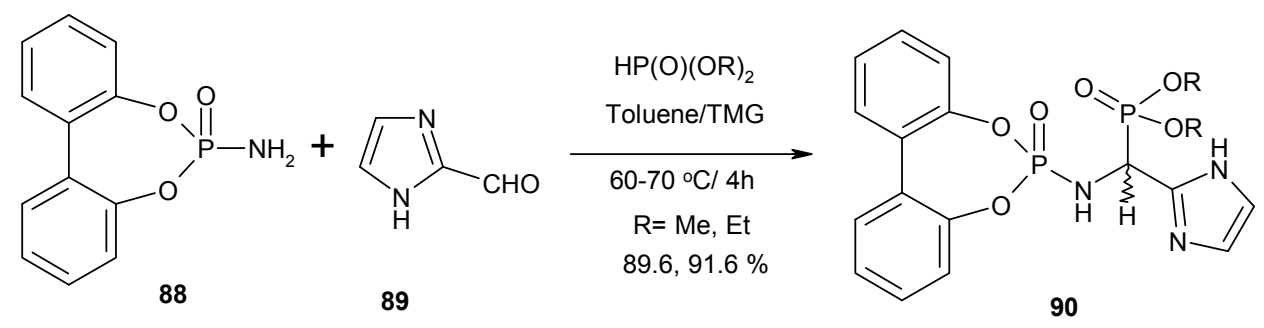

\section{Scheme 38}

Substituted $\alpha$-(benzodioxol-5-yl)- $\alpha$-aminophosphonates 91 could be prepared under various mild conditions by reaction of veratraldehyde, and dialkyl phosphites (Scheme 39). ${ }^{82-85}$

Three-component Mannich type reactions starting from aldehydes or ketones, amines and phosphites have proved to be a facile method for the preparation of various $\alpha$-aminoalkylphosphonate compounds. A rapid method for the synthesis of $N$-phosphoramino- $\alpha$ aminophosphonate 92 involved reacting veratraldehyde with diethyl phosphoramidate and a cyclic trivalent chlorophosphite at $50-60{ }^{\circ} \mathrm{C}$ neat, without solvent or catalyst, for an appropriate time (Scheme 40). ${ }^{86}$ 


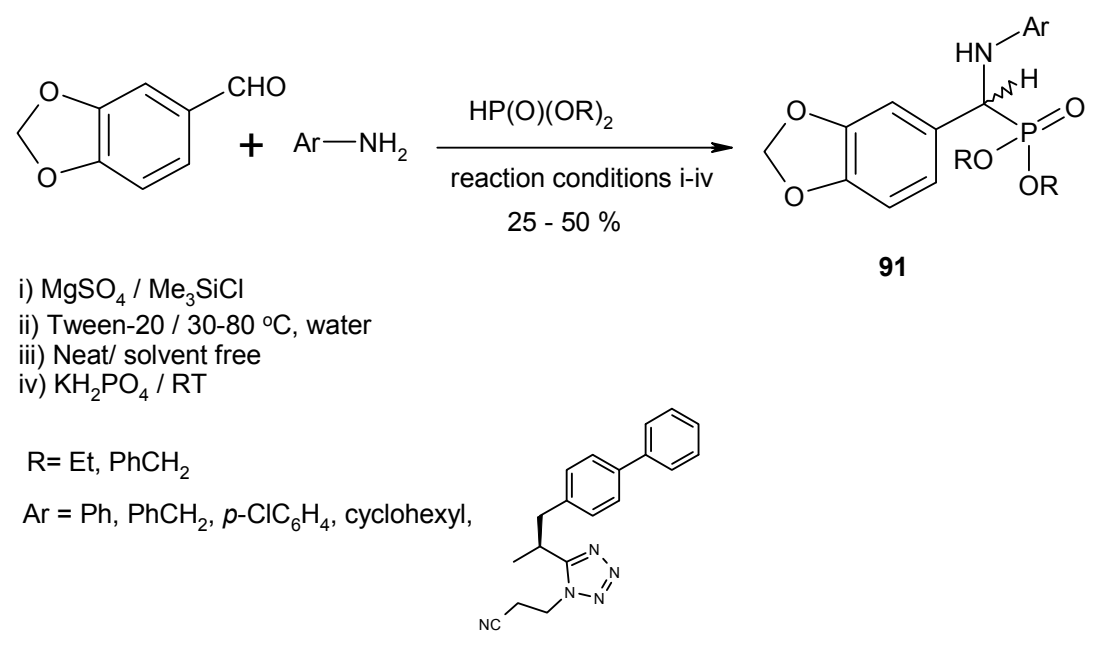

\section{Scheme 39}

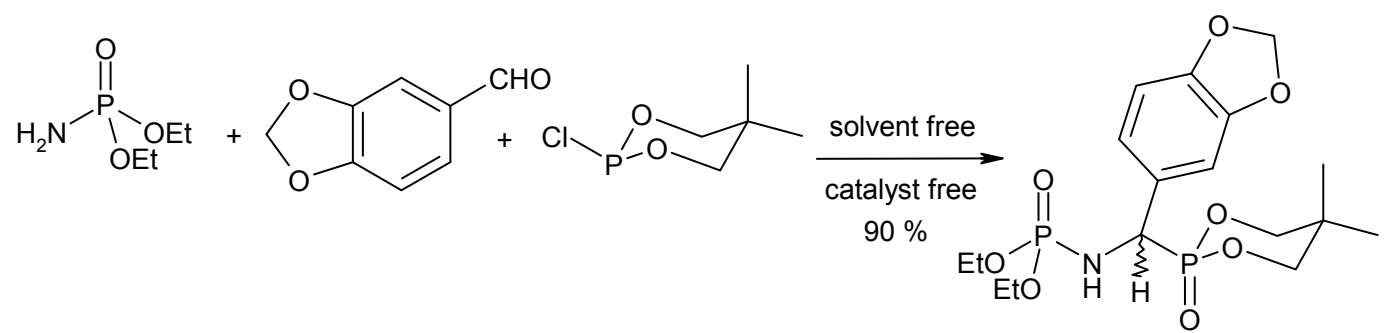

92

\section{Scheme 40}

Reaction between 2-formylbenzimidazole (93), 2-amino-5-methyloxazole (94), and diethyl phosphite by stirring equimolar quantities in a variety of solvents at ambient temperature gave a low yield of the desired $\alpha$-aminophosphonate 95 in all the experiments. The best result was obtained when the reaction was carried out using PEG in water (Scheme 41). ${ }^{74}$

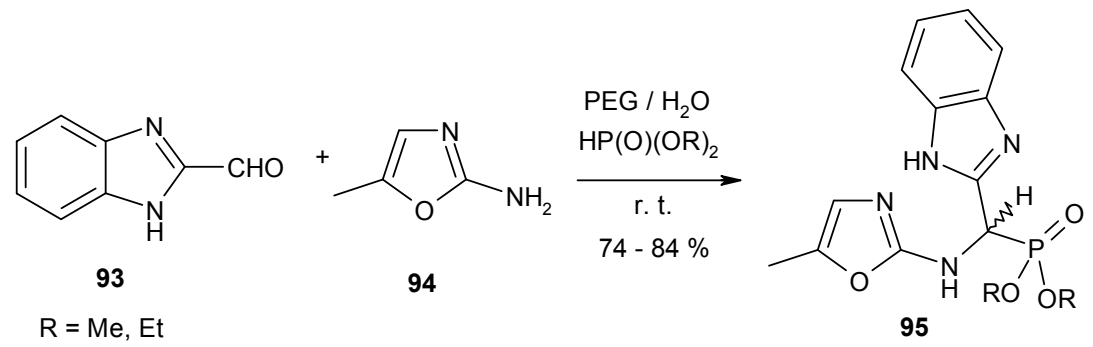

\section{Scheme 41}




\subsection{Six-membered heterocycles with one heteroatom}

The action of tris(trimethylsilyl)phosphite on the aldimine formed in situ from 2-formylchromone (96) and benzylamine yielded ( $N$-benzylamino)chromon-2-ylmethanephosphonic acid (97) (Scheme 42). ${ }^{87}$<smiles>O=Cc1cc(=O)c2ccccc2o1</smiles>

96<smiles>CO[C@H](c1ccccc1)[C@H](NCc1ccccc1)[C@H](NCc1ccccc1)P(=O)(O)O</smiles>

97

\section{Scheme 42}

3-Formylchromones (98) reacted easily with amines and dialkyl phosphites or trialkyl phosphites under different reaction conditions to form the $\alpha$-(chromon-3-yl)- $\alpha$-aminophosphonates 99 in moderate to high yields (Scheme 43). ${ }^{46,48}$

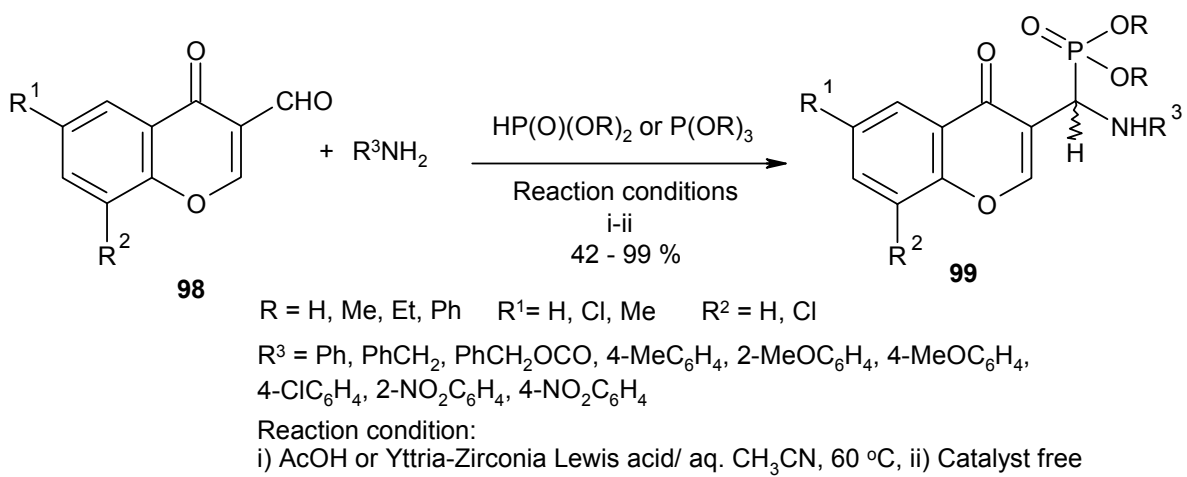

\section{Scheme 43}

The one pot three component reactions of heterocyclic ketones 100, amine and triethyl phosphite in ethanol or toluene/acetonitrile at $60{ }^{\circ} \mathrm{C}$ afforded $\alpha$-aminophosphonates $\mathbf{5 1}$ in low to good yields (Scheme 44). ${ }^{88}$

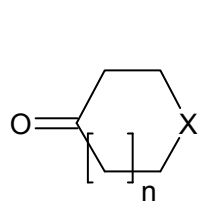

100
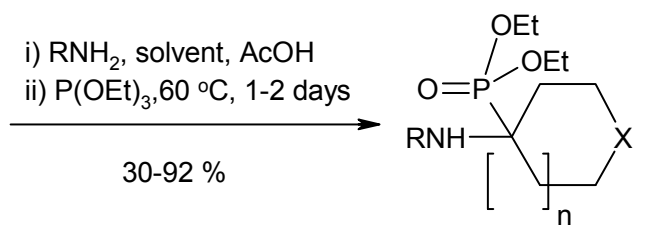

51

$\mathrm{X}=\mathrm{NMe}, \mathrm{O}, \mathrm{S}$

$\mathrm{RNH}=\mathrm{PhCH}\left(\mathrm{CH}_{3}\right) \mathrm{NH}_{2}, \mathrm{PhCH}_{2} \mathrm{NH}_{2}, \mathrm{PhCH}\left(\mathrm{CH}_{2} \mathrm{OH}\right) \mathrm{NH}_{2}$

Solvent $=\mathrm{EtOH}$, Toluene, Acetonitrile

\section{Scheme 44}


2-, 3-, And 4-formylpyridines 101 reacted with different alkyl and aromatic amines and trialkyl phosphite or dialkyl phosphite under different reaction conditions to give the corresponding $\alpha$-(pyridyl)- $\alpha$-aminophosphonates $\mathbf{1 0 2}$ in good to excellent yields (Scheme 45) (Table 4). ${ }^{10,52,58,59,61,66-68,71,72,84,89-93}$

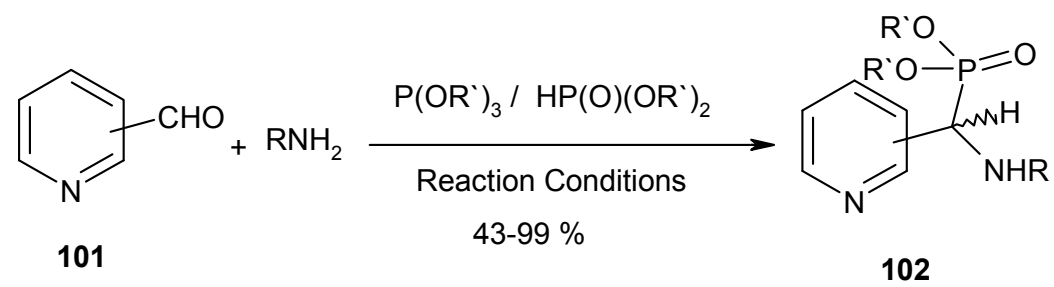

\section{Scheme 45}

Table 4. Reaction conditions of 2-, 3- and 4-formylpyridines 101 and amines with tri- or di-alkyl phosphite to give the corresponding pyridyl $\alpha$-aminophosphonates 102

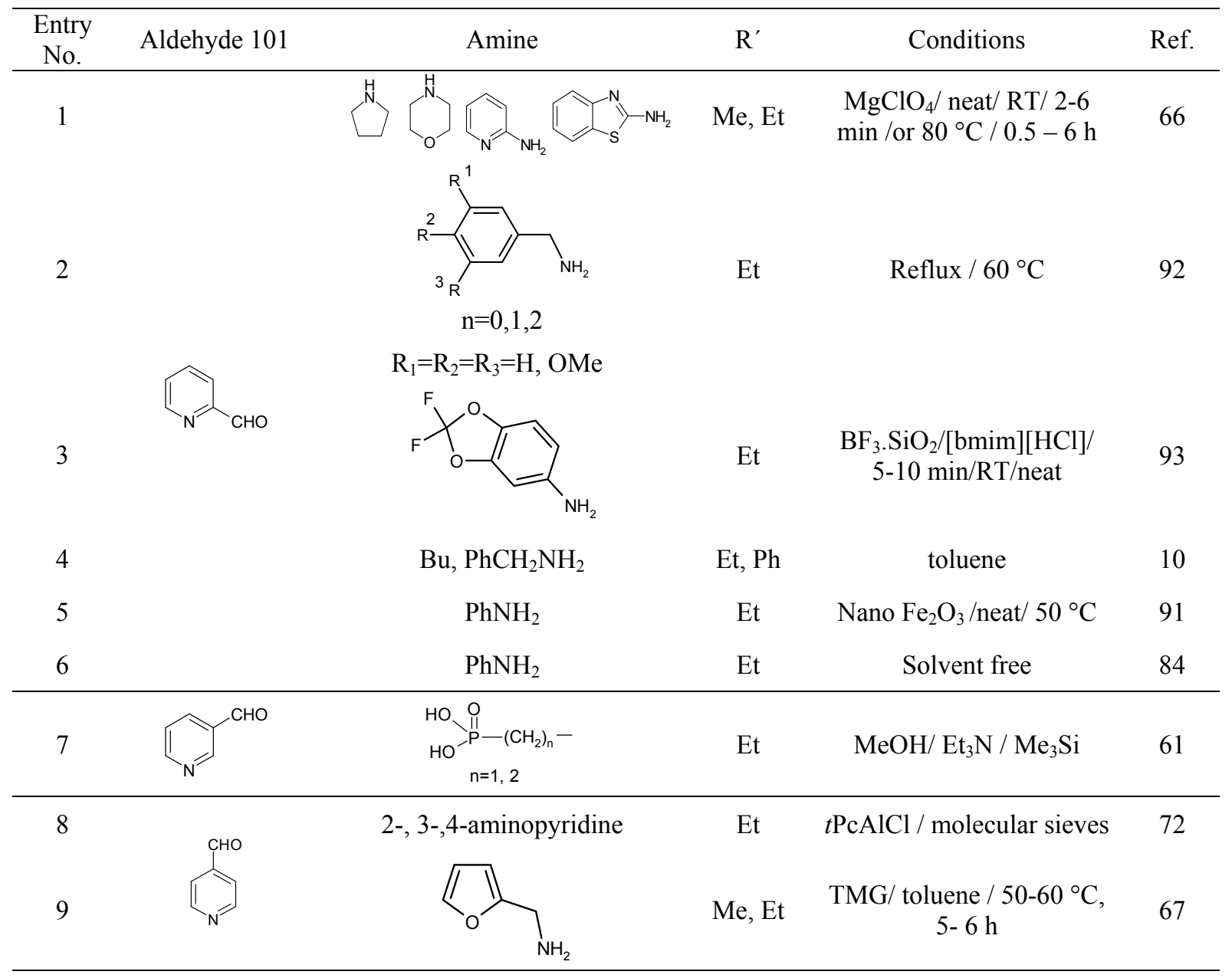


Table 4. Continued

\begin{tabular}{|c|c|c|c|c|c|}
\hline $\begin{array}{l}\text { Entry } \\
\text { No. }\end{array}$ & Aldehyde 101 & Amine & $\mathrm{R}^{\prime}$ & Conditions & Ref \\
\hline 10 & & $\left(\mathrm{Me}_{3} \mathrm{Si}\right)_{2} \mathrm{NH}$ & Et & $\begin{array}{c}\mathrm{Al}(\mathrm{OTf})_{3}(10 \mathrm{~mol} \%) / \\
\text { solvent free } / 80^{\circ} \mathrm{C}\end{array}$ & 59 \\
\hline 11 & & $\left(\mathrm{Me}_{3} \mathrm{Si}\right)_{2} \mathrm{NH}$ & Et & $\mathrm{I}_{2}(10 \mathrm{~mol} \%) /$ solvent free & 58 \\
\hline 12 & & $\mathrm{PhNH}_{2}$ & $\mathrm{Me}$ & $\mathrm{TiCl}_{4} / \mathrm{CH}_{3} \mathrm{CN} / \mathrm{RT}$ & 89 \\
\hline 13 & $\mathrm{CHO}$ & $\mathrm{PhNH}_{2}$ & $\mathrm{Me}$ & $\mathrm{AlCl}_{3} / \mathrm{CH}_{3} \mathrm{CN} / \mathrm{RT}$ & 52 \\
\hline 14 & & 2-aminopyridine & Et & Solvent free & 71 \\
\hline 15 & & & $\mathrm{Me}, \mathrm{Et}$ & toluene/TMG/ $60-70^{\circ} \mathrm{C}$ & 68 \\
\hline 16 & $\begin{array}{c}2-, 3-, 4- \\
\text { formylpyridines }\end{array}$ & $\begin{array}{c}\mathrm{BuNH}_{2}, \mathrm{PhNH}_{2}, \mathrm{Ph}_{2} \mathrm{CHNH}_{2}, \\
\mathrm{PhCH}_{2} \mathrm{NH}_{2}\end{array}$ & Et & toluene $/ 110^{\circ} \mathrm{C}$ & 90 \\
\hline
\end{tabular}

Reactions of various arylamines, 3-acetylpyridine and triphenyl phosphite in the presence of lithium perchlorate were carried out to give $\alpha$-(pyridine-3-yl)- $\alpha$-aminophosphonates 103 in high yields (Scheme 46). ${ }^{94}$

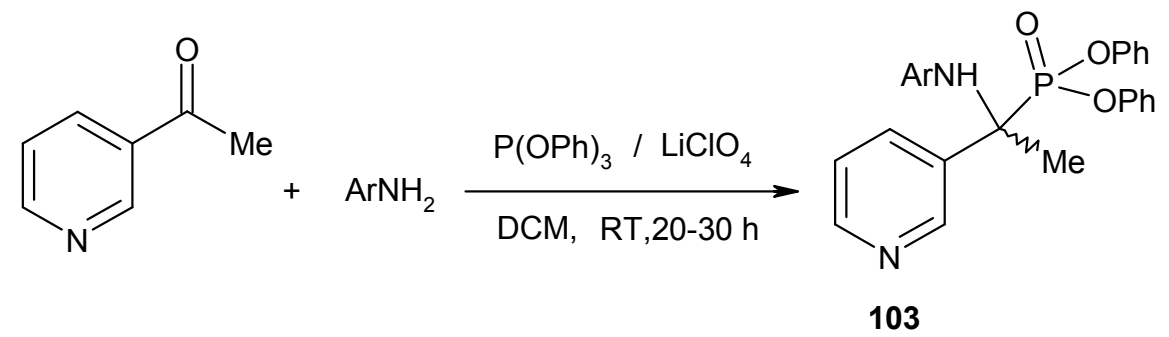

$$
\mathrm{Ar}=\mathrm{C}_{6} \mathrm{H}_{5}, 4-\mathrm{ClC}_{6} \mathrm{H}_{4}, 4-\mathrm{MeC}_{6} \mathrm{H}_{4}, 4-\mathrm{HOC}_{6} \mathrm{H}_{4}, \mathrm{NHC}_{6} \mathrm{H}_{5}
$$

\section{Scheme 46}

The three-component reaction of 4-(pyridin-4-yl)benzaldehyde and triethyl phosphite with various aryl/heteroaryl substituted primary amines led to the formation of diethyl (aryl/heteroaryl aminopyridine-4-yl)phenyl)methyl phosphonates 104 (Scheme 47). ${ }^{95}$

The interesting $\alpha$-quinonylmethylenyl $\alpha$-aminophosphonate 107 was prepared in moderate yield through reaction of 8-formylmethylquinoline 105, 3-[5-[(1S)-1-amino-2-(biphenyl-4-yl) ethyl]-1H-tetrazol-1-yl]propanenitrile (106) and dibenzyl phosphite in the presence of $\mathrm{MgSO}_{4}$ and $\mathrm{Me}_{3} \mathrm{SiCl}$ (Scheme 48). ${ }^{82}$ 


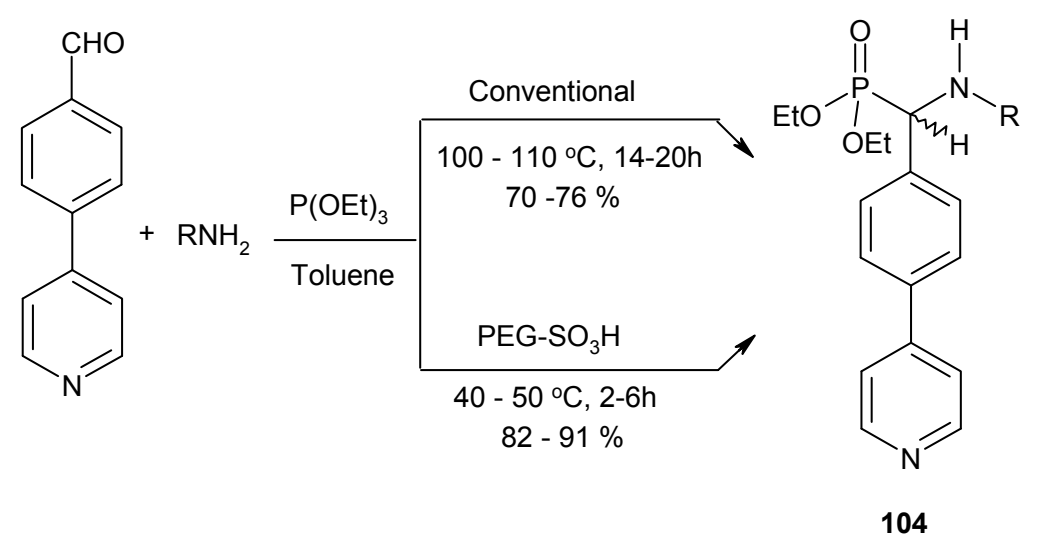

R= 4-pyridyl, 1-(pyridin-3-yl)methyl, 1,3-thiazol-2-yl, 1,3-benzothiazol-2-yl, 5-nitro-1,3-benzothiazol-2-yl

\section{Scheme 47}

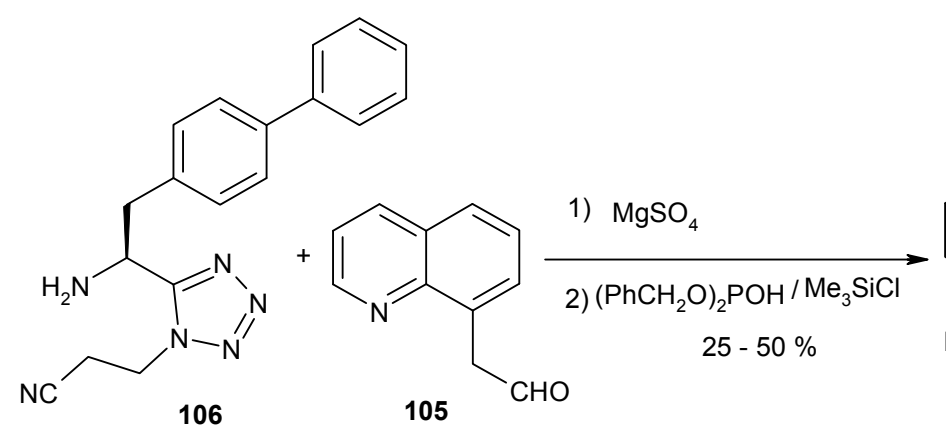

\section{Scheme 48}

One-pot three-component reaction of quinoline-3-carboxaldehyde and aniline with diethyl phosphite under microwave irradiation reaction proceeded in the formation of diethyl [ $\alpha$-anilino(3-quinolylmethyl)]phosphonate $\mathbf{1 0 8}$ in a relatively good yield of about $80 \%$. Unexpectedly, the corresponding monoester $109(3 \%)$ and a very interesting phosphorus compound that proved to be bis(hydrophosphonate) phosphate monoester derivative $110(7 \%)$ were isolated as byproducts (Scheme 49). ${ }^{96}$

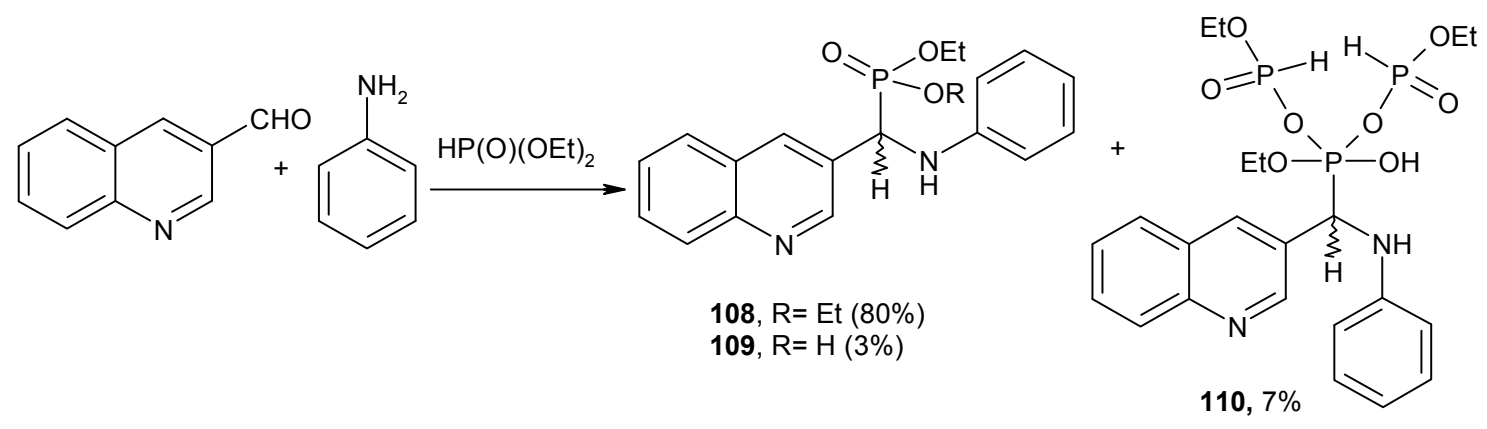

\section{Scheme 49}


A mixture of a 2-chloro-3-formylquinoline derivative 111, an amine and dialkyl phosphite and/or trialkyl phosphite was added to the solvent used under the reaction conditions shown to afford the corresponding $\alpha$-(quinolin-3-yl)- $\alpha$-aminophosphonates 112 in good to excellent yields (Scheme 50). ${ }^{42,48,85}$

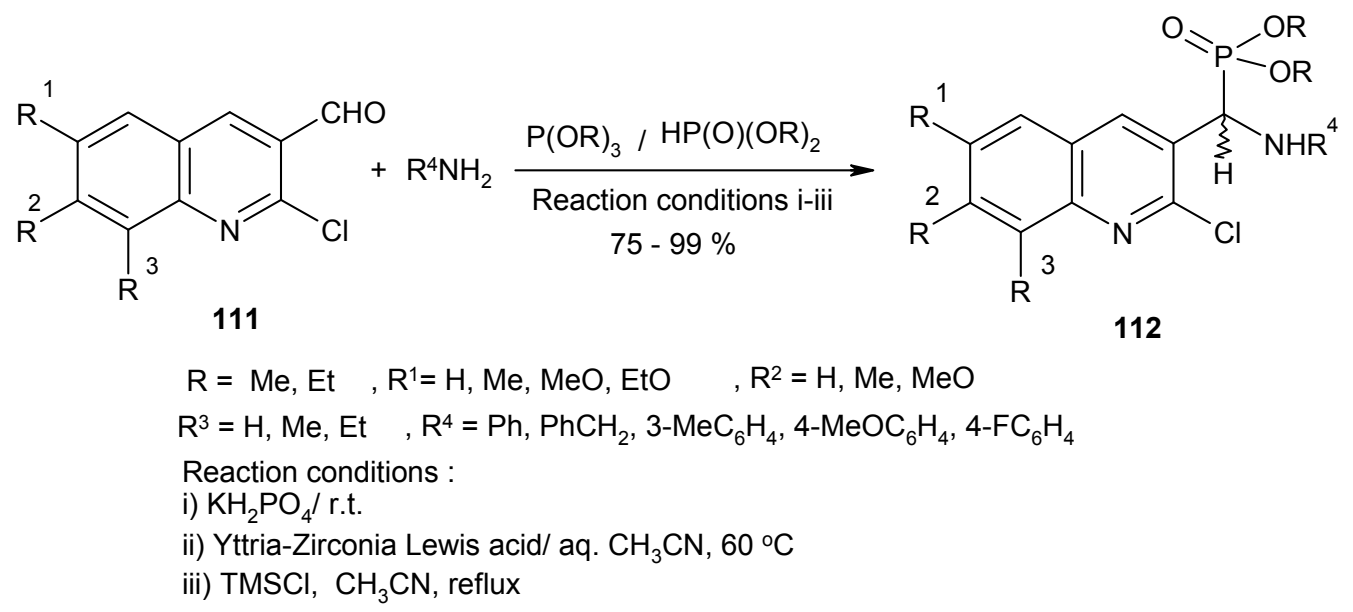

\section{Scheme 50}

\subsection{Six-membered heterocycles with two or more heteroatoms}

Hybrid compounds of $\alpha$-aminophosphonate 114 with pyrimidine nucleosides were synthesized in good to excellent yields starting from 5-formyl-2-deoxyuridine (113), aniline and dimethyl phosphite in one pot (Scheme 51). ${ }^{97,98}$

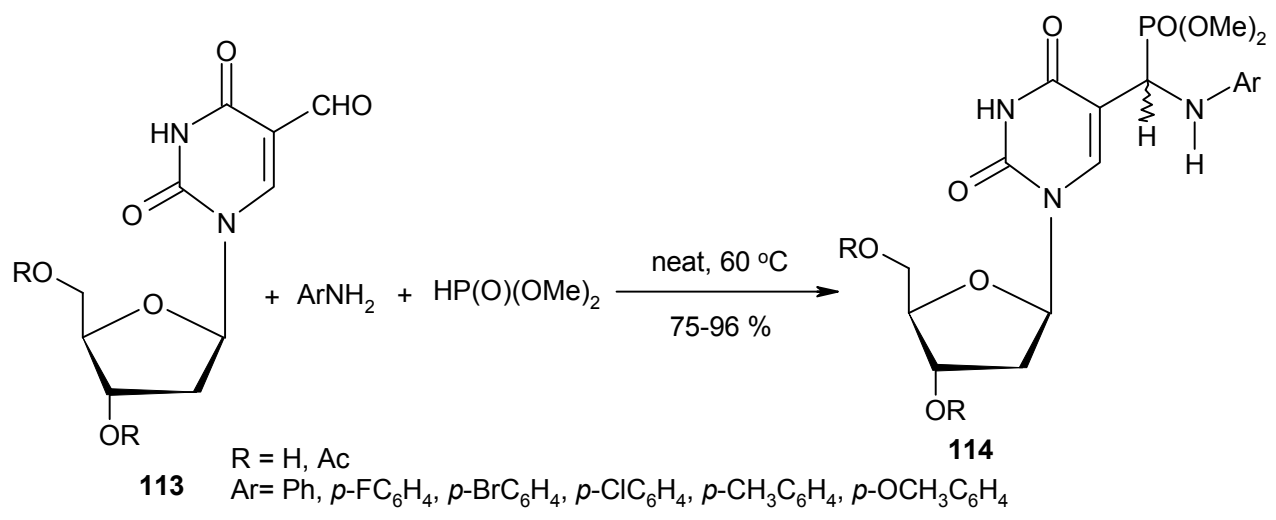

\section{Scheme 51}

The target $\alpha$-aminophosphonates 116 were synthesized via the Mannich-type reactions of aldehyde 115, aromatic amines, and dialkyl phosphites or triphenyl phosphite in the presence of $\mathrm{Mg}\left(\mathrm{ClO}_{4}\right)_{2}$ in moderate to good yields. It was found that $\mathrm{Mg}\left(\mathrm{ClO}_{4}\right)_{2}$ can reduce the reaction time and improve the yields of products greatly (Scheme 52). ${ }^{99}$ 

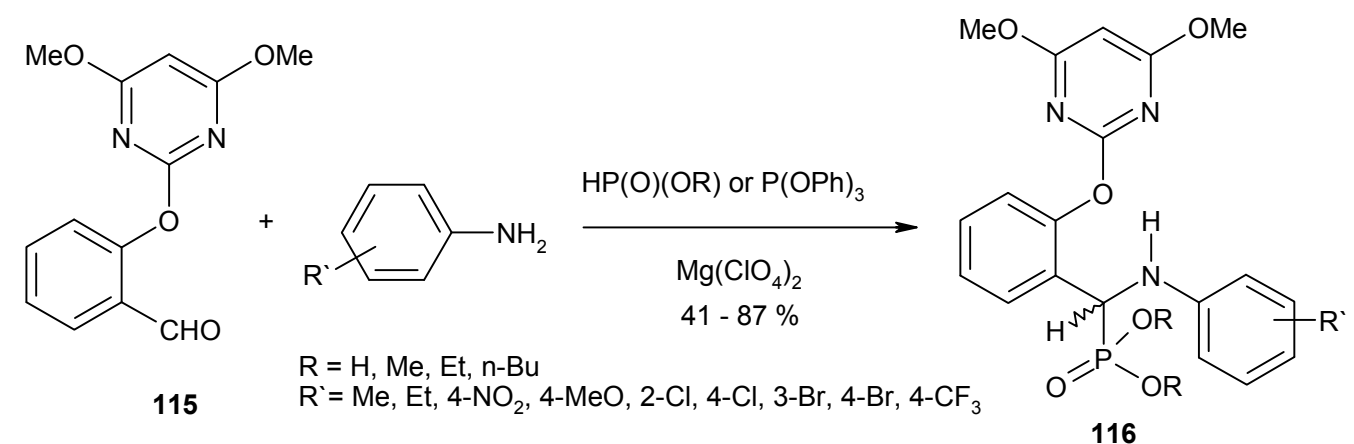

\section{Scheme 52}

The reaction of 10-ethyl-10H-phenothiazine-3-carbaldehyde 117, anilines, and diethyl phosphite in PEG-400 was complete in 24 hour at room temperature; the corresponding $\alpha$ aminophosphonates 118 was obtained in low yield (30\%). However, the yield was dramatically increased by increasing the temperature to $100{ }^{\circ} \mathrm{C}$. Under optimized conditions, the reaction proceeded well at $100{ }^{\circ} \mathrm{C}$ and the desired $\alpha$-aminophosphonate $118(\mathrm{Ar}=\mathrm{Ph})$ was obtained in 91\% yield. PEG-400 was found to be more effective in the synthesis of $118(\mathrm{Ar}=\mathrm{Ph})$ in terms of reaction time $(6 \mathrm{~h})$ and yields $(91 \%)$ (Scheme 53$).{ }^{100}$

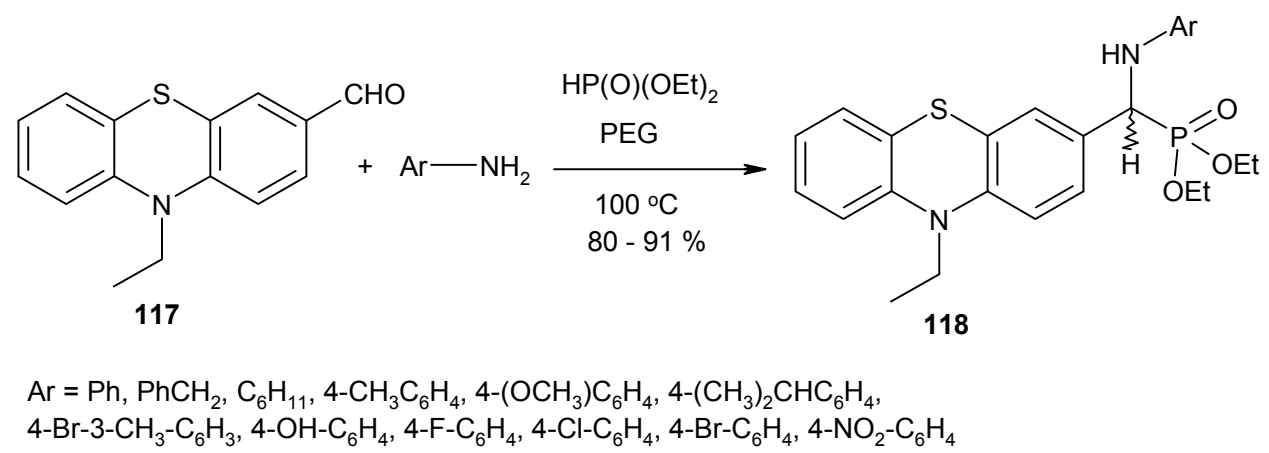

\section{Scheme 53}

\subsection{Macrocycles}

$\alpha$-Aminophosphonic acid derivatives of benzo-15-crown-5-ether 120 can be easily obtained from the 4-formylbenzo-15-crown-5 (119), primary amines, and trimethyl phosphite or diethyl phosphite. The aminophosphonic acid $\mathbf{1 2 0}$ was obtained as a free base by using bromotrimethylsilane as a deprotecting agent of phosphonic esters (Scheme 54). ${ }^{101}$ 

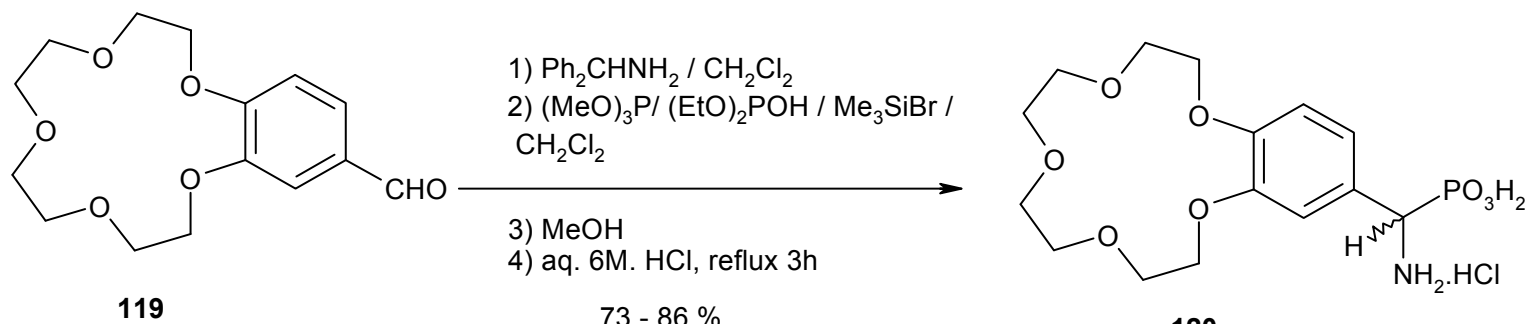

120

\section{Scheme 54}

\section{Miscellaneous Methods}

\subsection{From diethyl $\alpha$-azido- $\alpha$-(benzoylaminomethyl)phosphonate}

Also, diethyl (2-benzoylamino-2-(4,5-dicarboxymethyl-1,2,3-triazol-1-yl)-methyl) phosphonate (122) was prepared by a reaction of diethyl-(2-azido-2-benzoylaminomethyl) phosphonate (121) with dimethyl acetylenedicarboxylate in dry benzene at room temperature (Scheme 55). ${ }^{102}$

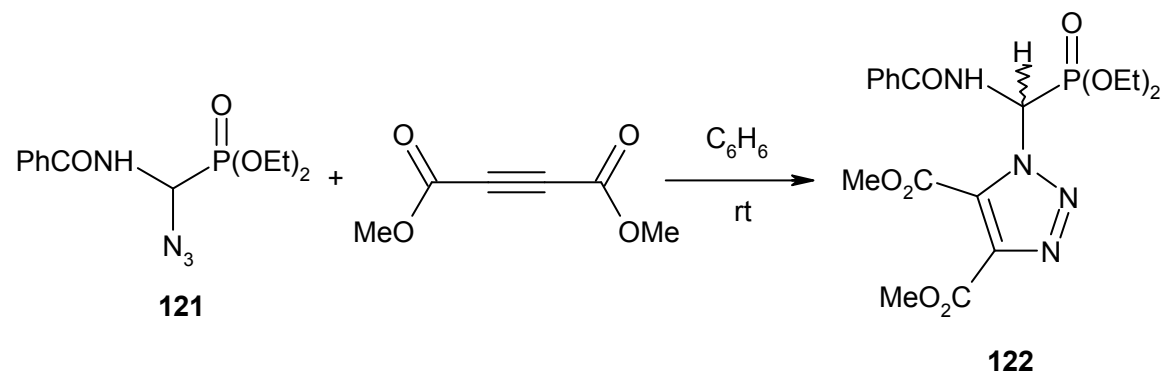

\section{Scheme 55}

Similarly, some 1-(prop-2-ynyl)-5-aryltetrazoles (123) and 2-(prop-2-ynyl)-5-aryltetrazoles (124) were submitted to undergo cycloaddition reaction with the azido phosphonate $\mathbf{1 2 1}$ in dry benzene under reflux to give the interesting biheterocyclic $\alpha$-aminophosphonic acid diesters $\mathbf{1 2 5}$, 126 and 127, 128, respectively (Scheme 56). ${ }^{103}$ 


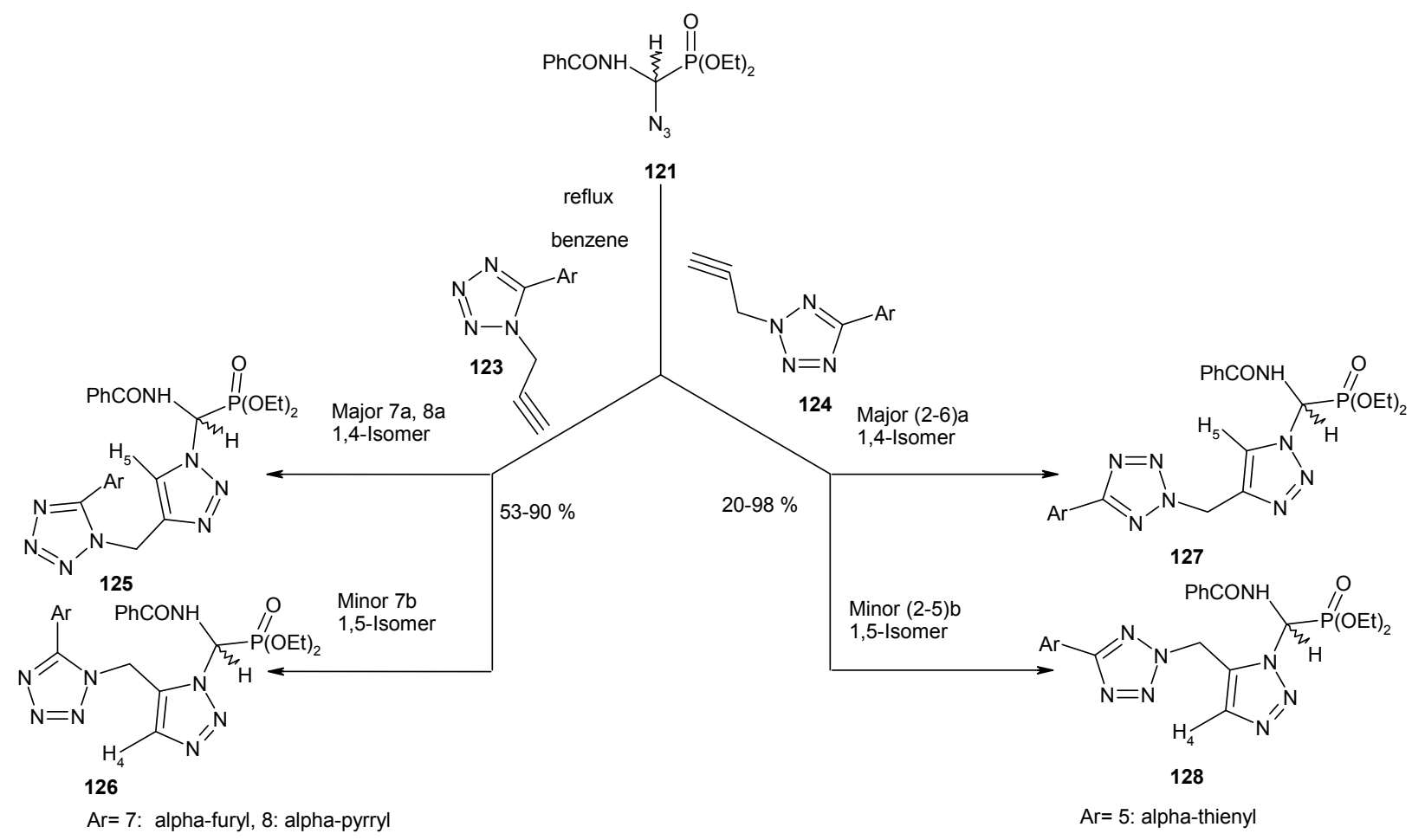

\section{Scheme 56}

\subsection{Nucleophilic substitution reactions}

The reactions of different heterocyclic nucleophiles (benzimidazole, imidazole, pyrazole, 3,5dimethylpyrazole and 1,2,4-triazole) with diethyl $\alpha$-azido- $\alpha$-aminomethylphosphonate (121) were conducted at room temperature in acetone in the presence of DIPEA (diisopropylethylamine) resulting in the corresponding $\alpha$-heterocyclic $\alpha$-aminophosphonates 129 (Scheme 57). ${ }^{104}$

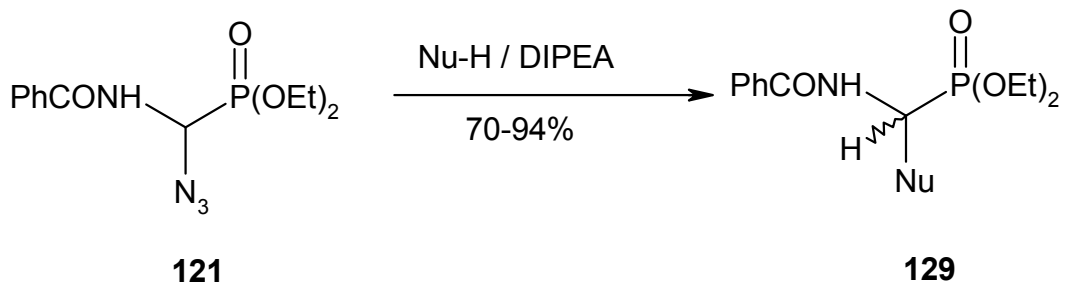

$\mathrm{Nu}-\mathrm{H}=$ Benzimidazole, Imidazole, Pyrazole, 3,5-Dimethylpyrazole, 1,2,4-Triazole

\section{Scheme 57}

Nucleophilic substitution of trichloromethyl moiety in ureidophosphonate $\mathbf{1 3 0}$ with morpholine gave diethyl $\{1$-[3-(2-hydroxyphenyl)ureido]-2-morpholino-2-oxoethyl $\}$ phosphonate (131) in $82 \%$ yield (Scheme 58). ${ }^{105}$ 


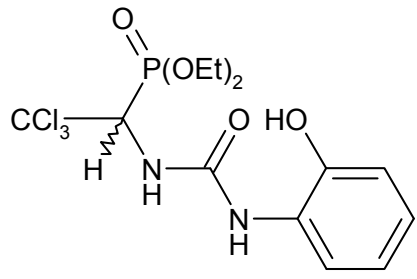

130

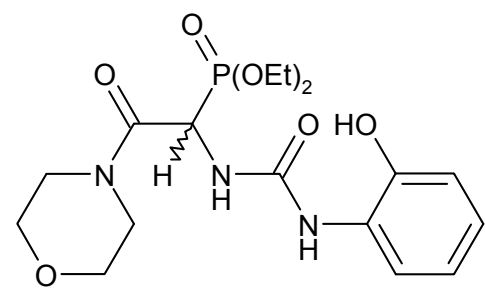

131

\section{Scheme 58}

Similarly, the reaction of different amines $\mathrm{Nu}-\mathrm{H}$ with the $\alpha$-bromo- $\alpha$-aminophosphonate derivative 132 resulted in the formation of $\alpha$-heterocyclic $\alpha$-aminophosphonates $\mathbf{1 3 3}$ (Scheme 59). ${ }^{106}$
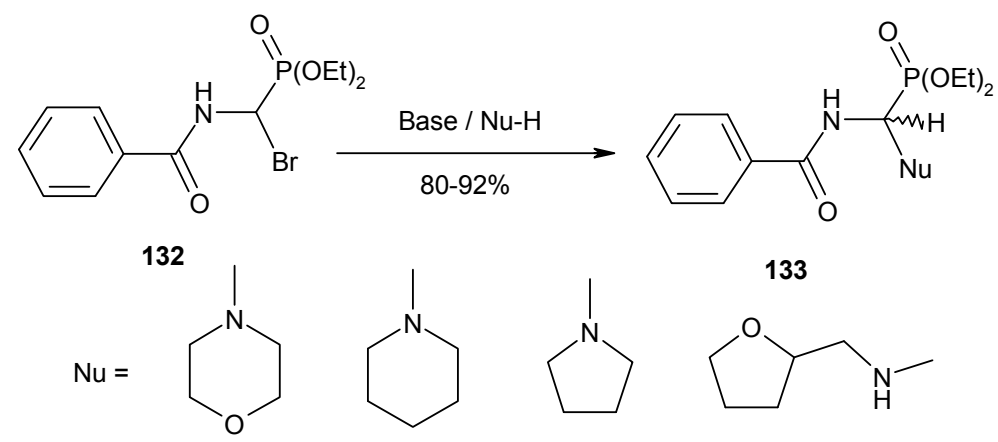

\section{Scheme 59}

\subsection{Cycloadditions of $\alpha$-alkynylaminophosphonates}

The cycloaddition of diethyl [(1-[(benzyloxycarbonyl)amino]-1-(trifluoromethyl)prop/but-2-yn1-yl]phosphonate (134) to organic azides proceeded only at $80{ }^{\circ} \mathrm{C}$ in the presence of a solvent mixture to afford the corresponding $\alpha-\mathrm{CF}_{3}-\alpha$-(triazol-4-yl)- $\alpha$-aminophosphonates 135 (Scheme 60). ${ }^{107}$

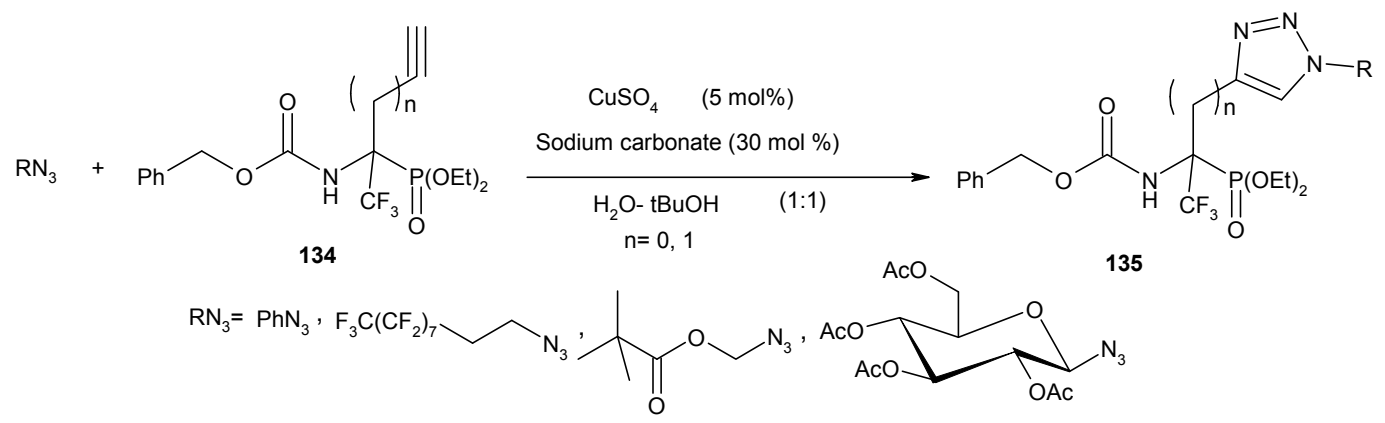

\section{Scheme 60}


Also, the ethynyl-substituted aminophosphonate 134 demonstrated comparable reactivity towards different nitrile oxides under similar reaction conditions, yielding the corresponding isoxazole-containing $\alpha$-trifluoromethyl $\alpha$-aminophosphonates $\mathbf{1 3 6}$ in good yield (Scheme 61). ${ }^{108}$

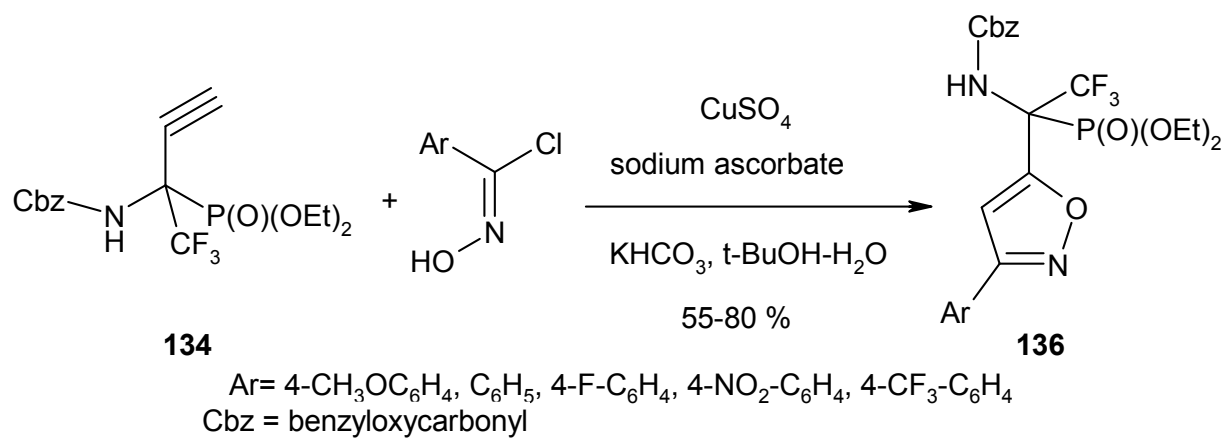

\section{Scheme 61}

\subsection{Reduction of $\alpha$-hydroxyimino phosphonate}

Simple reduction of the 1-hydroxyiminophosphonate 137 with $\mathrm{NaBH}_{4}$ in the presence of transition metal compounds such as $\mathrm{MoO}_{3}$ or $\mathrm{NiCl}_{2} \cdot 6 \mathrm{H}_{2} \mathrm{O}$ at ambient temperature in methanol and at normal pressure gave the corresponding diethyl aminoalkyl(aryl)phosphonates $\mathbf{1 3 8}$ in good yields (Scheme 62). ${ }^{109}$

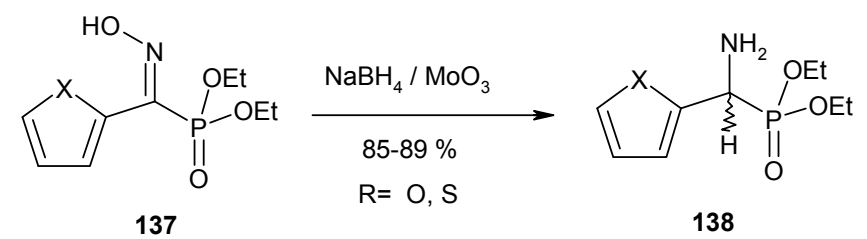

\section{Scheme 62}

\subsection{Hydrolysis of $S$-adenosyl-L-homocysteine derivative}

Reaction of the thiolate anion of 139 with diethyl [3-bromo-1-(diphenylmethyleneamino)propyl] phosphonate (140) was performed to give the fully protected AdoHcy analogue 141 in 63\% yield. Deprotection was achieved via hydrolysis with trimethylsilyl iodide (TMSI) to generate the desired $\alpha$-aminophosphonic acid nucleoside 142 in 75\% yield (Scheme 63). ${ }^{110}$ 


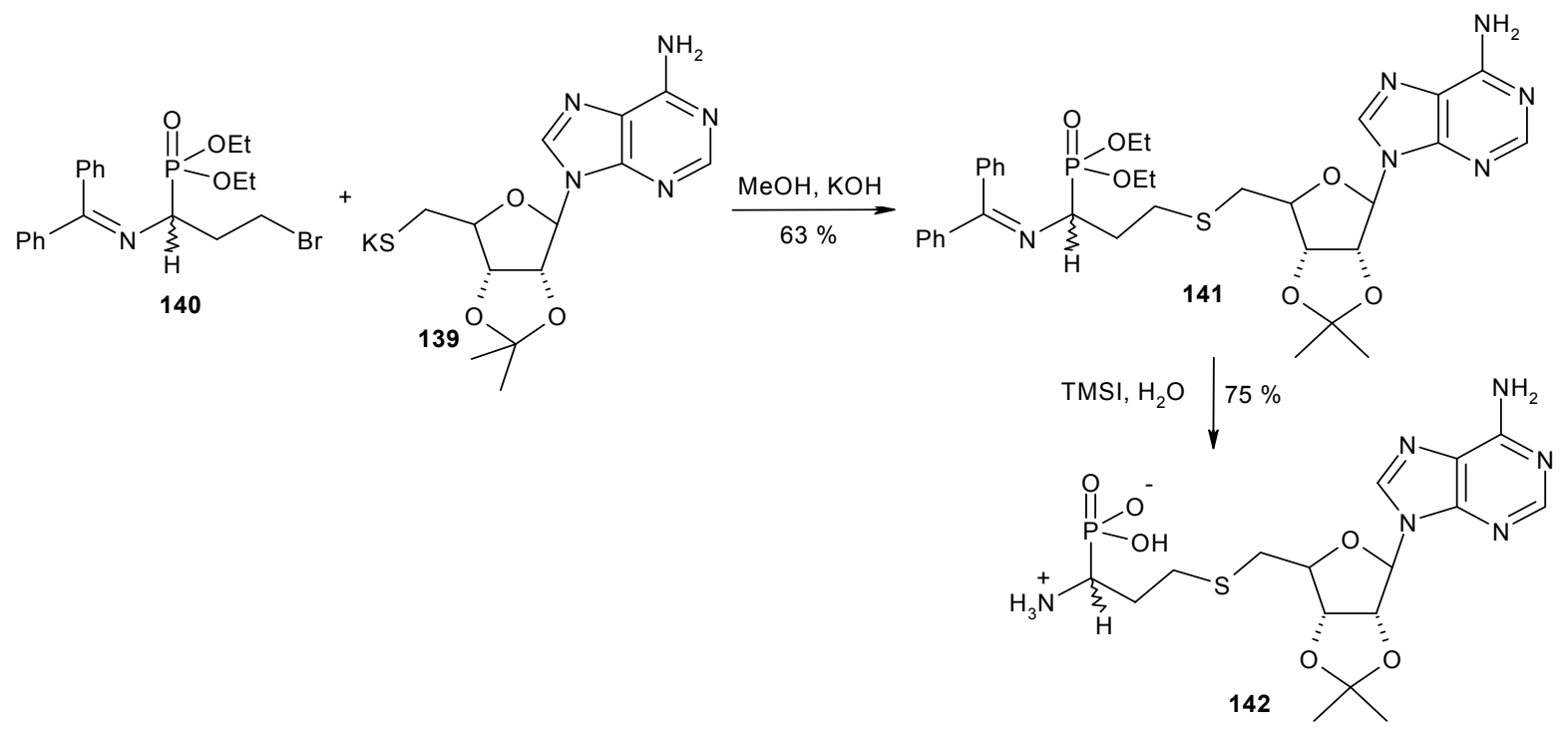

\section{Scheme 63}

\subsection{Curtius rearrangement of $\alpha$-acylazidophosphonate}

Curtius rearrangement of the $S, S$-dioxide of $\alpha$-acylazidophosphonate 143 in dichloromethane afforded $\alpha$-Boc- $\alpha$-aminophosphonate 144 (Scheme 64). ${ }^{111}$

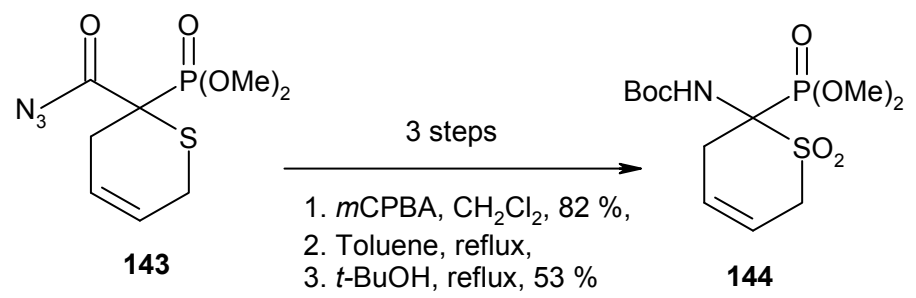

\section{Scheme 64}

\subsection{Addition of diethyl phosphite to chiral N-benzyl nitrones}

When N,N-diprotected $\alpha$-aminonitrones 145 were treated with TBDMSOTf and then diethyl phosphite in THF or $\mathrm{CH}_{2} \mathrm{Cl}_{2}$ at $-20{ }^{\circ} \mathrm{C}$, the syn $\alpha$-(hydroxyamino)phosphonates 146 were obtained in good yields after flash chromatography (Scheme 65). ${ }^{112}$ 

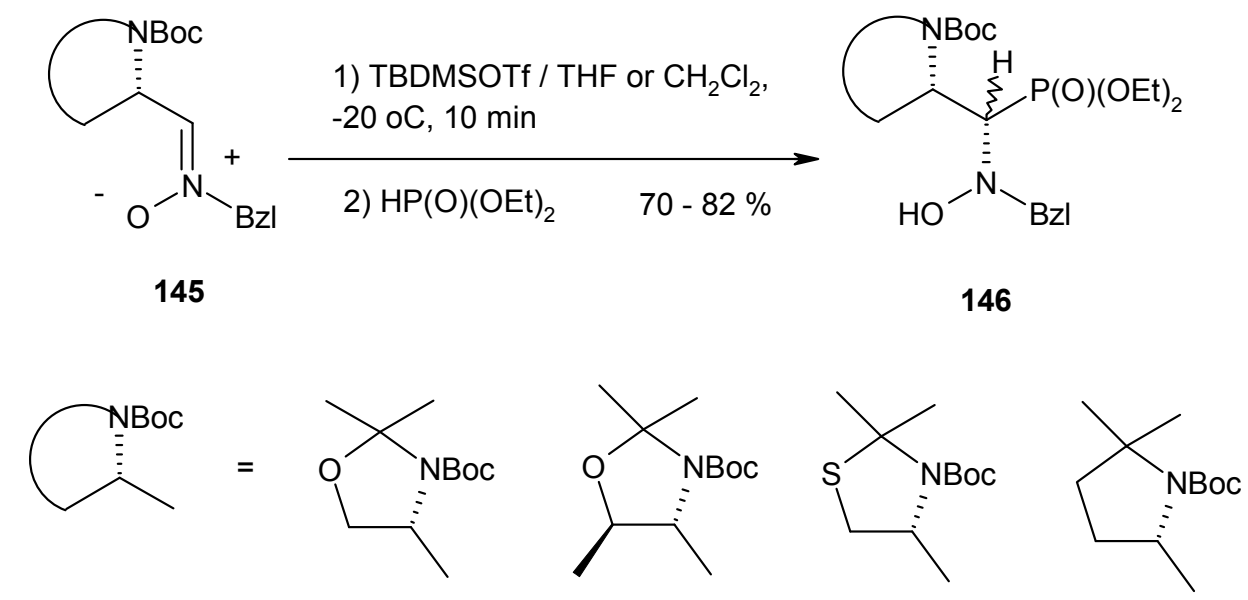

\section{Scheme 65}

\subsection{From phosphonyliminium salts}

The optimized reaction conditions involved refluxing of the phosphonyliminium salt 147 and some nucleophilic aromatic compounds in THF gave the highest yields of novel $\alpha-$ heteroaryl- $\alpha$-aminophosphonates 151 (Scheme 66). ${ }^{113}$

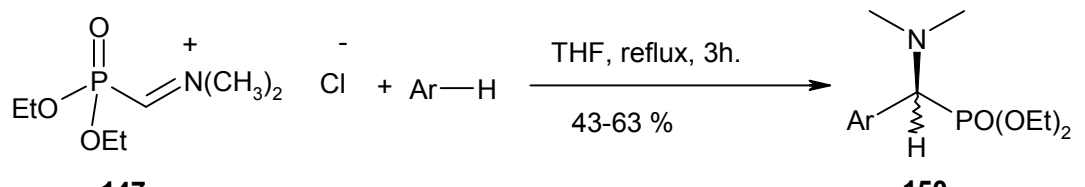

147<smiles>Cc1cn(C)c2ccccc12</smiles><smiles>Cc1cccn1C</smiles><smiles>Cc1ccc(C)o1</smiles>

\section{Scheme 66}

\subsection{From oxazolyl phosphonates}

Heating diethyl 5-(2-acylhydrazino)-2-[(4-methylphenyl)-1,3-oxazol-4-yl]phosphonates 151 in acetic acid led to the formation of the phosphonic acid derivatives $\mathbf{1 5 2}$ through ring opening and recyclization of the oxazole derivative and ester hydrolysis (Scheme 67). ${ }^{114}$ 


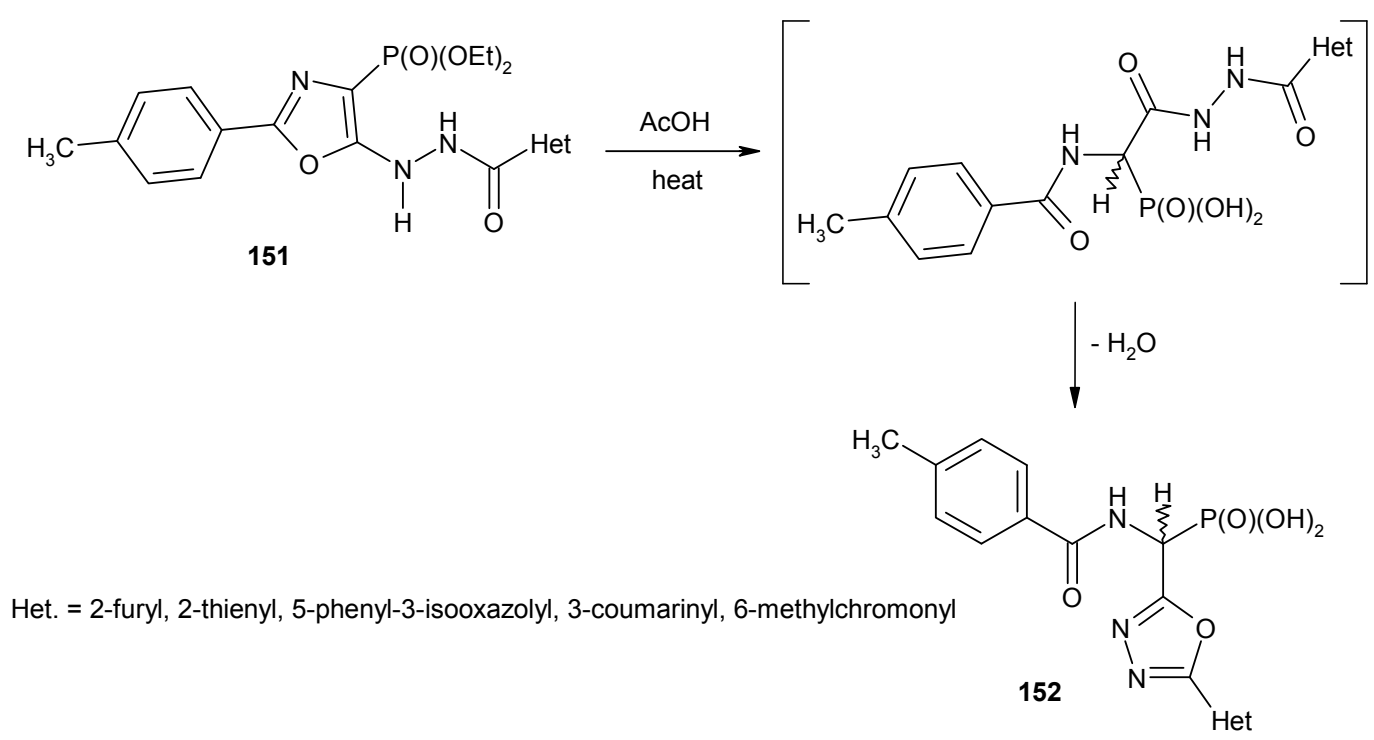

\section{Scheme 67}

Finally, reaction of the oxazolyl phosphonates 151 (Het=6-methylchromone) with hydrazine hydrate in ethanol for 2 hours afforded diethyl [5-[5-(2-hydroxyphenyl/2-hydroxy-5methylphenyl)-1H-pyrazol-3-yl]-1,3,4-oxadiazol-2-yl][(4-methylbenzoyl)amino]methylphosphonates 153 in good yields, which were boiled in acetic acid to afford the corresponding phosphonic acid derivatives 154 (Scheme 68). ${ }^{114}$

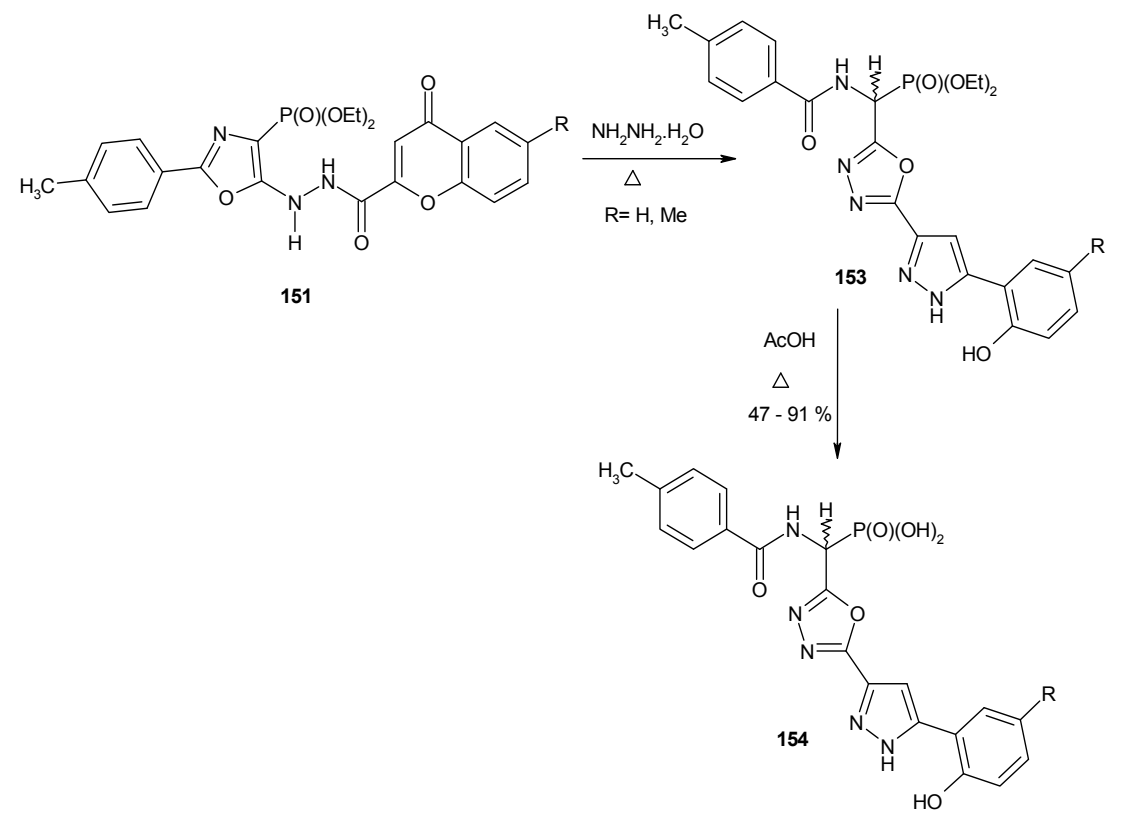

\section{Scheme 68}




\section{Conclusions}

During the last few years, the $\alpha$-aminophosphonic acids have attracted considerable attention in the scientific community and a great variety of methodologies have been reported for the synthesis of these compounds. The importance of having new relevant structures has allowed the development of new strategies and synthetic procedures. The authors of this review have collected the most relevant procedures reported up to the end 2013 on the synthesis of $\alpha$ heterocyclic/heteroaryl $\alpha$-aminophosphonic acids and their esters that will be a fundamental key in the design of new bioactive agents with improved pharmacological properties. The review is built up according to the used methods and starting with the smallest rings of each method.

\section{References}

1. Saito, J. B.; Egami, H.; Katsuki, T. J. Am. Chem. Soc. 2007, 129, 1978. http://dx.doi.org/10.1021/ja0651005

2. Kukhar, V. P.; Hudson, H. R. Aminophosphonic and Aminophosphinic Acids: Chemistry and biological activity. John Wiley. Chichester. 2000.

3. Treov, K. D. Chemistry and application of H-phosphonates. Elsevier, Amsterdam 2006.

4. Kafarski, P.; Lejczak, B. Phosphorus, Sulfur Silicon Relat. Elem. 1991, 63, 193. http://dx.doi.org/10.1080/10426509108029443

5. Kaplan, A. P.; Bartlett, P. A. Biochemistry 1991, $30,8165$. http://dx.doi.org/10.1021/bi00247a011

6. Atherton, F. R.; Hassall, C. H.; Lambert, R. W. J. Med. Chem. 1986, 29, 29. http://dx.doi.org/10.1021/jm00151a005

7. De Lombaert, S.; Blanchard, L.; Tan, T.; Sakane, Y.; Berry, C.; Ghai, R. D. Bioorg. Med. Chem. Lett. 1995, 5, 145. http://dx.doi.org/10.1016/0960-894X(94)00474-T

8. Kafarski, P.; Lejczak, B. Current Med. Chem. Anti-Cancer Agents 2001, 1, 301. http://dx.doi.org/10.2174/1568011013354543

9. Emsley, J.; Hall, D. The Chemistry of Phosphorus. Harper and Row, London, 1976.

10. Boduszek, B. Phosphorus, Sulfur Silicon Relat. Elem. 1999, 144, 433. http://dx.doi.org/10.1080/10426509908546274

11. Jacobsen, N. E.; Bartlett, P. A. J. Am. Chem. Soc. 1981, 103, 654. http://dx.doi.org/10.1021/ja00393a026

12. De Risi, C.; Perrone, D.; Dondoni, A.; Pollini, G. P.; Bertolasi, V. Eur. J. Org. Chem. 2003, 1904.

13. (a) Yokomatsu, T.; Yoshida, Y.; Shibuya, S. J. Org. Chem. 1994, 59, 7930. http://dx.doi.org/10.1021/jo00104a064

(b) Ordonez, M.; Rojas-Cabera, H.; Cativiela, C. Tetrahedron 2009, 65, 17. 
http://dx.doi.org/10.1016/j.tet.2008.09.083

(c) Kudzin, Z. H.; Kudzin, M. H.; Drabowicz, J.; Stevens, C. V. Curr. Org. Chem. 2011, $15,2015$.

http://dx.doi.org/10.2174/138527211795703612

(d) Ordonez, M.; Sayago, F. J.; Cativiela, C. Tetrahedron 2012, 68, 6369.

http://dx.doi.org/10.1016/j.tet.2012.05.008

14. (a) Chandrasekhar, S.; Narsihmulu, C.; Sultana, S. S.; Saritha, B.; Jayaprakash, S. Synlett 2003, 4, 505 .

http://dx.doi.org/10.1055/s-2003-37508

(b) Agawane, S. M.; Nagarkar, J. M. Tetrahedron Lett. 2011, 52, 3499.

http://dx.doi.org/10.1016/j.tetlet.2011.04.112

(c) Ranu, B. C.; Hajra, A. Green Chem. 2002, 4, 551.

15. (a) Chandrasekhar, S.; Prakash, S. J.; Jagadeshwar, V.; Narsihmulu, C. Tetrahedron Lett. 2001, 42, 5561 .

http://dx.doi.org/10.1016/S0040-4039(01)01053-X

(b) Dake, S. A.; Raut, D. S.; Kharat, K. R.; Mhaske, R. S.; Deshmukh, S. U.; Pawar, R. P. Bioorg. Med. Chem. Lett. 2011, 21, 2527;

http://dx.doi.org/10.1016/j.bmcl.2011.02.039

(c) Balint, E.; Takacs, J.; Drahos, L.; Juranovic, A.; Kocevar, M.; Keglevich, G. Heteroatom. Chem. 2013, 24, 221;

http://dx.doi.org/10.1002/hc.21086

(d) Reddy, M. V. N.; Kumar, B. S.; Balakrishna, A.; Reddy, C. S.; Nayak, S. K.; Reddy, C.

D. Arkivoc 2007, xv, 246.

http://dx.doi.org/10.3998/ark.5550190.0008.f24

16. Ali, T. E. Arkivoc 2014, (i), 21.

17. Ali, T. E.; Halacheva, S. S. Heteroatom. Chem. 2009, 20, 117.

http://dx.doi.org/10.1002/hc.20520

18. Ali, T. E. Phosphorus, Sulfur Silicon Relat. Elem. 2010, 185, 88.

http://dx.doi.org/10.1080/10426500802713309

19. Ali, T. E. Eur. J. Med. Chem. 2009, 44, 4539.

http://dx.doi.org/10.1016/j.ejmech.2009.06.022

20. Bouduszek, B. Phosphorus, Sulfur Silicon Relat. Elem. 1995, 104, 63. http://dx.doi.org/10.1080/10426509508042578

21. Skowronski, R.; Grabowski, G.; Nazarski, R. B.; Lewkowski, J. A. Phosphorus, Sulfur Silicon Relat. Elem. 1999, 144-146, 449.

22. Nazarski, R. B.; Lewkowski, J. 1. A.; Skowronski, R. Heteroatom Chem. 2002, 13, 120. http://dx.doi.org/10.1002/hc.10005

23. Hubert, C.; Garrigues, B.; Munoz, A. Phosphorus, Sulfur Silicon Relat. Elem. 1999, 152, 229.

http://dx.doi.org/10.1080/10426509908031632 
24. Oussaid, A.; Benyaqad, F.; Oussaid, B.; Pradel, C.; Garriguesa, B. Phosphorus, Sulfur Silicon Relat. Elem. 2003, 178, 1183.

http://dx.doi.org/10.1080/10426500307903

25. Hudson, H. R.; Lee, R. J.; Matthews, R. W. Phosphorus, Sulfur Silicon Relat. Elem. 2004, $179,1691$.

http://dx.doi.org/10.1080/10426500490466274

26. Claeys, D. D.; Moonen, K.; Roman, B. I.; Nemykin, V. N.; Zhdankin, V. V.; Waroquier, M.; Van Speybroeck, V.; Stevens, C. V. J. Org. Chem. 2008, 73, 7921.

http://dx.doi.org/10.1021/jo801138s

27. Louaisil, N.; Rabasso, N.; Fadel, A. Tetrahedron 2009, 65, 8587.

http://dx.doi.org/10.1016/j.tet.2009.07.040

28. Kraicheva, I. Phosphorus, Sulfur Silicon Relat. Elem. 1999, 155, 127. http://dx.doi.org/10.1080/10426509908044976

29. Kraicheva, I.; Finocchiaro, P.; Failla, S. Phosphorus, Sulfur Silicon Relat. Elem. 2002, 177, 2915.

http://dx.doi.org/10.1080/10426500214891

30. Kraicheva, I.; Finocchiaro, P.; Failla, S. Phosphorus, Sulfur Silicon Relat. Elem. 2004, 179, 2345.

http://dx.doi.org/10.1080/10426500490485093

31. Kraicheva, I.; Finocchiaro, P.; Failla, S. Phosphorus, Sulfur Silicon Relat. Elem. 2007, 182, 57.

http://dx.doi.org/10.1080/10426500600866903

32. Kraicheva, I.; Bogomilova, A.; Tsacheva, I.; Momekov, G.; Momekova, D.; Troev, K. Eur. J. Med. Chem. 2010, 45, 6039.

http://dx.doi.org/10.1016/j.ejmech.2010.10.002

33. Salkeeva, L. K.; Nurmaganbetova, M. T.; Kurmanaliev, O. S.; Gazizov, T. K. Russ. J. Org. Chem. 2002, 38, 723.

http://dx.doi.org/10.1023/A:1019671324617

34. Krishna, A. B.; Reddy, M. V. N.; Reddy, G. C. S.; Krishna, B. S.; Nayak, S. K.; Reddy, C. S. Inter. J. Appl. Bio. Pharm. Tech. 2010, I, 873.

35. Olszewski, T. K.; Boduszek, B.; Sobek, S.; Kozłowski, H. Tetrahedron 2006, 62, 2183. http://dx.doi.org/10.1016/j.tet.2005.12.016

36. Pyrkosz, M.; Goldeman, W.; Kontecka, E. G. Inorg. Chim. Acta 2012, 380, 223. http://dx.doi.org/10.1016/j.ica.2011.09.024

37. Boduszek, B.; Olszewski, T. K.; Goldeman, W.; Grzegolec, K.; Blazejewska, P. Tetrahedron 2012, 68, 1223. http://dx.doi.org/10.1016/j.tet.2011.11.054

38. Menard, L.; Fontaine, L.; Brosse, J. C. React. Polym. 1994, 23, 201. http://dx.doi.org/10.1016/0923-1137(94)90022-1 
39. Boduszek, B.; Korenova, A.; Uher, M.; Vegh, D. Phosphorus, Sulfur Silicon Relat. Elem. 2003, 178, 1047.

http://dx.doi.org/10.1080/10426500307846

40. Mandhane, P. G.; Joshi, R. S.; Nagargoje, D. R.; Chate, A. V.; Gill, C. H. Phosphorus, Sulfur Silicon Relat. Elem. 2011, 186, 149.

http://dx.doi.org/10.1080/10426507.2010.492363

41. Pokalwar, R. U.; Sadaphal, S. A.; Kategaonkar, A. H.; Shingate, B. B.; Shingare, M. S. Green Chem. Lett. Rev. 2010, 3, 33. http://dx.doi.org/10.1080/17518250903410082

42. Pokalwar, R. U.; Hangarge, R. V.; Madje, B. R.; Ware, M. N.; Shingare, M. S. Phosphorus, Sulfur Silicon Relat. Elem. 2008, 183, 1461.

http://dx.doi.org/10.1080/10426500701681532

43. Michalska, J.; Boduszek, B.; Olszewski, T. K. Heteroatom Chem. 2011, 22, 617. http://dx.doi.org/10.1002/hc.20704

44. Kategaonkar, A. H.; Sonar, S. S.; Sapkal, S. B.; Gawali, V. U.; Shingate, B. B.; Shingare, M. S. Phosphorus, Sulfur Silicon Relat. Elem. 2010, 185, 2113. http://dx.doi.org/10.1080/10426500903530867

45. Khidre, M. D.; Abou-Yousef, H. M.; Mahran, M. R. H. Phosphorus, Sulfur Silicon Relat. Elem. 1998, 140, 147. http://dx.doi.org/10.1080/10426509808035740

46. Lang, S. Y.; Wang, D. Z.; Cao, L. H. Phosphorus, Sulfur Silicon Relat. Elem. 2009, 184, 2560 .

http://dx.doi.org/10.1080/10426500802529127

47. Ali, T. E. Arkivoc 2008, (ii), 71.

48. Ramalingam, S.; Kumar, P. Catal Lett. 2008, 125, 315. http://dx.doi.org/10.1007/s10562-008-9562-x

49. Sadaphal, S. A.; Kategaonkar, A. H.; Sapkal, S. B.; Shingate, B. B.; Gill, C. H.; Shingare, M. S. Bull. Cat. Soc. India 2009, 8, 131.

50. Sadaphal, S. A.; Sonar, S. S.; Kategaonkar, A. H.; Shingare, M. S. Bull. Korean Chem. Soc. 2009, 30, 1054.

http://dx.doi.org/10.5012/bkes.2009.30.5.1054

51. Niralwad, K. S.; Shingate, B. B.; Shingare, M. S. Ultrasonics Sonochem. 2010, 17, 760. http://dx.doi.org/10.1016/j.ultsonch.2010.02.002

52. Manjula, A.; Rao, B. V.; Neelakantan, P. Synth. Commun. 2003, 33, 2963. http://dx.doi.org/10.1081/SCC-120022468

53. Sobhani, S.; Safaei, E.; Asadi, M.; Jalili, F. J. Organometal. Chem. 2008, 693, 3313. http://dx.doi.org/10.1016/j.jorganchem.2008.07.037

54. Yadav, J. S.; Reddy, B. V. S.; Sreedhar, P. Green Chem. 2002, 4, 436. http://dx.doi.org/10.1039/b203934f

55. Sun, P.; Hu, Z.; Huang, Z. Synth. Commun. 2004, 34, 4293. 


\section{http://dx.doi.org/10.1081/SCC-200039361}

56. El Kaim, L.; Grimaud, L.; Hadrot, S. Tetrahedron Lett. 2006, 47, 3945.

http://dx.doi.org/10.1016/j.tetlet.2006.03.151

57. Ohara, M.; Nakamura, S.; Shibataa, N. Adv. Synth. Catal. 2011, 353, 3285.

http://dx.doi.org/10.1002/adsc.201100482

58. Sobhani, S.; Vafaee, A. J. Iran. Chem. Soc. 2010, 7, 227.

59. Sobhani, S.; Tashrifi, Z. Heteroatom Chem. 2009, 20, 109.

http://dx.doi.org/10.1002/hc.20517

60. Tillu, V. H.; Dumbre, D. K.; Wakharkar, R. D.; Choudhary, V. R. Tetrahedron Lett. 2011, 52,863 .

http://dx.doi.org/10.1016/j.tetlet.2010.11.105

61. Zhang, X. Y.; Qu, Y. Y.; Fan, X. S. Phosphorus, Sulfur Silicon Relat. Elem. 2010, 185, 816.

http://dx.doi.org/10.1080/10426500902994338

62. Sarvari, M. H. Tetrahedron 2008, 64, 5459.

http://dx.doi.org/10.1016/j.tet.2008.04.016

63. Agawane, S. M.; Nagarkar, J. M. Tetrahedron Lett. 2011, 52, 3499. http://dx.doi.org/10.1016/j.tetlet.2011.04.112

64. Sheykhan, M.; Ma'mani, L.; Ebrahimi, A.; Heydari, A. J. Mol. Catal. A: Chem.2011, 335, 253.

http://dx.doi.org/10.1016/j.molcata.2010.12.004

65. Bai, S.; Song, B.; Bhadury, P. S.; Yang, S.; Hu, D.; Xue, W. Chin. J. Chem. 2011, 29, 109. http://dx.doi.org/10.1002/cjoc.201190035

66. Bhagat, S.; Chakraborti, A. K. J. Org. Chem. 2007, 72, 1263.

http://dx.doi.org/10.1021/jo062140i

67. Reddy, M. V. N.; Annar, S.; Krishna, A. B.; Reddy, G. C. S.; Reddy, C. S. Org. Commun. 2010, 3, 39.

68. Reddy, M. V. N.; Balakrishna, A.; Kumar, M. A.; Reddy, G. C. S.; Sankar, A. U. R.; Reddy, C. S.; Krishna, T. M. Chem. Pharm. Bull. 2009, 57, 1391.

http://dx.doi.org/10.1248/cpb.57.1391

69. Kaboudin, B.; Afsharinezhad, M. B.; Yokomatsu, T. Arkivoc 2012, (iv), 44.

70. Siemieniec, J.; Kafarski, P.; Plucinski, P. Molecules 2013, 18, 8473. http://dx.doi.org/10.3390/molecules18078473

71. Zefirov, N. S.; Matveeva, E. D. Arkivoc 2008, (i), 1. http://dx.doi.org/10.3998/ark.5550190.0009.101

72. Matveeva, E. D.; Podrugina, T. A.; Kolesnikova, I. N.; Borisenko, A. A.; Zefirov, N. S. Russ. Chem. Bull. Int. Ed. 2009, 58, 119.

73. Sobhania, S.; Tashrifia, Z. Synth. Commun. 2009, 39, 120. http://dx.doi.org/10.1080/00397910802369695 
74. Rao, K. U. M.; Jayaprakash, S. H.; Nayak, S. K.; Reddy, C. S. Catal. Sci. Technol. 2011, 1, 1665 .

http://dx.doi.org/10.1039/c1cy00295c

75. Reddy, G. S.; Rao, K. U. M.; Sundar, C. S.; Sudha, S. S.; Haritha, B.; Swapna, S.; Reddy, C. S. Arabian J. Chem. 2014, 7, 833-838. http://dx.doi.org/10.1016/j.arabjc.2013.01.004

76. Mungara, A. K.; Park, Y. K.; Lee, K. D. Chem. Pharm. Bull. 2012, 60, 1531.

77. Ali, T. E. Heteroatom Chem. 2013, 24, 426.

http://dx.doi.org/10.1002/hc.21110

78. Lang, S. Y.; Yue, F.; Cao, L. H. Phosphorus, Sulfur Silicon Relat. Elem. 2012 , 187, 461. http://dx.doi.org/10.1080/10426507.2011.625510

79. Abdel-Megeed, M. F.; Badr, B. E.; Azaam, M. M.; El-Hiti, G. A. Phosphorus, Sulfur Silicon Relat. Elem. 2012, 187, 1462.

http://dx.doi.org/10.1080/10426507.2012.690117

80. Qiu, J.; Liu, G.; Li, J.; Zhao, Y.; Li, H.; Zhang, G. Chin. J. Org. Chem. 2011, 31, 63. http://dx.doi.org/10.1080/10426507.2012.690117

81. Abdel-Aziz, S. A.; Ali, T. E.; El-Mahdy, K. M.; Abdel-Karim, S. M. Eur. J. Chem. 2011, 2, 25.

http://dx.doi.org/10.5155/eurjchem.2.1.25-35.208

82. Lombaert, S. D.; Stamford, L. B.; Blanchard, L.; Tan, J.; Hoyer, D.; Diefenbacher, C. G.; Wei, D.; Wallace, E. M.; Moskal, M. A., Savage, P.; Jeng, A. Y. Bioorg. Med. Chem. Lett. 1997, 7, 1059.

http://dx.doi.org/10.1016/S0960-894X(97)00159-5

83. Sundar, C. S.; Srinivasulu, D.; Nayak, S. K.; Reddy, C. S. Phosphorus, Sulfur Silicon Relat. Elem. 2012, 187, 523. http://dx.doi.org/10.1080/10426507.2011.631641

84. Ranu, B. C.; Hajra, A. Green Chem. 2002, 4, 551. http://dx.doi.org/10.1039/b205747f

85. Joshi, R. S.; Mandhane, P. G.; Dabhade, S. K.; Gill, C. H. Green Chem. Lett. Rev. 2010, 3, 191.

http://dx.doi.org/10.1080/17518251003660147

86. Zhang, J.; Cui, Z.; Wang, F.; Wang, Y.; Miao, Z.; Chen, R. Green Chem. 2007, 9, 1341. http://dx.doi.org/10.1039/b710008f

87. Boduszek, B.; Llpfiskt, M.; Kowalska, M. Phosphorus, Sulfur Silicon Relat. Elem. 1998, 143, 179. http://dx.doi.org/10.1080/10426509808045496

88. Rabasso, N.; Louaisil, N.; Fadel, A. Tetrahedron 2006, 62, 7445. http://dx.doi.org/10.1016/j.tet.2006.05.016

89. Reddy, Y. T., Reddy, P. N., Kumar, B. S., Rajput, P., Sreenivasulu, N., Rajitha, B. Phosphorus, Sulfur Silicon Relat. Elem. 2007, 182, 161. 
http://dx.doi.org/10.1080/10426500600887461

90. Boduszek, B. Tetrahedron 1996, 52, 12483.

http://dx.doi.org/10.1016/0040-4020(96)00727-2

91. Reddy, B. V. S.; Krishna, A. S.; Ganesh, A. V.; Kumar, G. G. K. S. N. Tetrahedron Lett. 2011, 52, 1359.

http://dx.doi.org/10.1016/j.tetlet.2011.01.074

92. Huang, K.-B.; Chen, Z.-F.; Liu, Y.-C.; Li, Z.-Q.; Wei, J.-H.; Wang, M.; Xie, X.-L.; Liang, H. Eur. J. Med. Chem. 2013, 64, 554.

http://dx.doi.org/10.1016/j.ejmech.2013.04.024

93. Reddy, M. V.; Dindulkar, S. D.; Jeong, Y. T. Tetrahedron Lett. 2011, 52, 4764. http://dx.doi.org/10.1016/j.tetlet.2011.07.027

94. Abdel-Megeed, M. F.; Badr, B. E.; Azaam, M. M.; El-Hiti, G. A. Bioorg. Med. Chem. 2012, 20, 2252.

http://dx.doi.org/10.1016/j.bmc.2012.02.015

95. Reddy, C. B.; Kumar, K. S.; Kumar, M. A.; Reddy, M. V. N.; Krishna, B. S.; Naveen, M.; Arunasree, M. K.; Reddy, C. S.; Raju, C. N.; Reddy, C. D. Eur. J. Med. Chem. 2012, 47, 553.

http://dx.doi.org/10.1016/j.ejmech.2011.11.026

96. Juribasic, M.; Stella, L.; Marinic, E.; Vinkovic, M.; Traldi, P.; Tuek-Bozic, L. Lett. Org. Chem. 2009, 6, 11.

http://dx.doi.org/10.2174/157017809787003142

97. Zhang, X. Y.; Qu, Y. Y.; Fan, X. S. Chin. Chem. Lett. 2010, 21, 1191. http://dx.doi.org/10.1016/j.cclet.2010.04.037

98. Zhang, X.; Qu, Y.; Fan, X.; Bores, C.; Feng, D.; Andrei, G.; Snoeck, R.; Clercq, E. D.; Loiseau, P. M. Nucleosides, Nucleotides Nucleic Acids, 2010, 29, 616. http://dx.doi.org/10.1080/15257770.2010.496281

99. Tang, W.; Yu, Z. H.; Shi, D. Q. Heteroatom Chem. 2010, 21, 148. http://dx.doi.org/10.1002/hc.20589

100. Kumar, M. A.; Lee, K. D. Phosphorus, Sulfur Silicon Relat. Elem. 2012, 187, 899. http://dx.doi.org/10.1080/10426507.2011.645174

101. Boduszeka, B.; Luboch, E. Phosphorus, Sulfur Silicon Relat. Elem. 2004, 179, 2527. http://dx.doi.org/10.1080/10426500490485543

102. Achamlale, S.; Mabrouk, H.; Elachqar, A.; El Hallaoui, A.; El Hajji, S.; Alami, A.; Bellan, J.; Mazières, M. R.; Wolf, J. G.; Pierrot, M. Phosphorus, Sulfur Silicon Relat. Elem. 2007, $182,357$. http://dx.doi.org/10.1080/10426500600919959

103. Achamlale, S.; Elachqar, A.; El Hallaoui, A.; Alami, A.; Elhajji, S.; Roumestant, M. L.; Viallefont, P. H. Phosphorus, Sulfur Silicon Relat. Elem. 1998, 140, 103. http://dx.doi.org/10.1080/10426509808035736 
104. Boukallaba, K.; Elachqar, A.; El Hallaoui, A.; Alami, A.; El Hajji, S.; Labriti, B.; Martinez, J.; Rolland, V. Phosphorus, Sulfur Silicon Relat. Elem. 2006, 181, 819.

http://dx.doi.org/10.1080/10426500500272046

105. Kockritz, A.; Schnell, M. Phosphorus, Sulfur Silicon Relat. Elem. 1993, 83, 125. http://dx.doi.org/10.1080/10426509308034355

106. Boukallaba, K.; Elachqar, A.; El Hallaoui, A.; Alami, A.; El Hajji, S.; Labriti, B.; Atmani, A.; El Bali, B.; Lachkar, M.; Allouchi, H.; Martinez, J.; Rolland, V. Phosphorus, Sulfur Silicon Relat. Elem. 2007, 182, 1045.

http://dx.doi.org/10.1080/10426500601093739

107. Vorobyeva, D. V.; Karimova, N. M.; Vasilyeva, T. P.; Osipov, S. N.; Shchetnikov, G. T.; Odinets, I. L.; Schenthaler, G. R. J. Fluorine Chem. 2010, 131, 378.

http://dx.doi.org/10.1016/j.jfluchem.2009.12.003

108. Vorobyeva, D. V.; Karimova, N. M.; Odinets, I. L.; Roschenthaler, G. V.; Osipov, S. N.; Org. Biomol. Chem. 2011, 9, 7335.

http://dx.doi.org/10.1039/c1ob06040f

109. Demir, A. S.; Tanyeli, C.; Sesenoglu, O.; Demic, S.; Evin, O. O. Tetrahedron Lett. 1996, $37,407$. http://dx.doi.org/10.1016/0040-4039(95)02171-X

110. Steere, J. A.; Sampson, P. B.; Honek, J. F. Bioorg. Med. Chem. Lett. 2002, 12, 457. http://dx.doi.org/10.1016/S0960-894X(01)00789-2

111. Moore, J. D.; Sprott, K. T.; Hanson, P. R. J. Org. Chem. 2002, 67, 8123. http://dx.doi.org/10.1021/jo0262208

112. Risi, C. D.; Perrone, D.; Dondoni, A.; Pollini, G. P.; Bertolasi, V. Eur. J. Org. Chem. 2003, 1904.

113. Risch, N.; Piper, S.; Winter, A.; Risse, A. L. Eur. J. Org. Chem. 2005, 387. http://dx.doi.org/10.1002/ejoc.200400618

114. Golovchenko, A. V.; Solomyannyi, R. N.; Brovarets, V. S. Russ. J. Gen. Chem. 2010, 80, 723. http://dx.doi.org/10.1134/S1070363210040067

\section{Authors' Biographies}

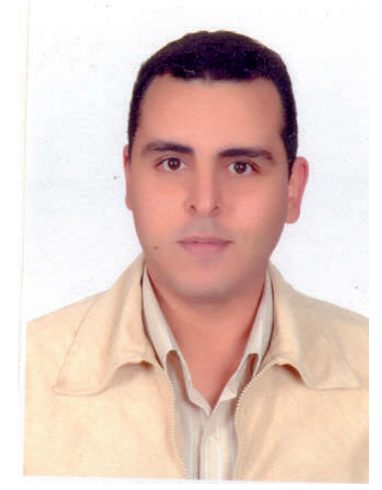


Tarik El-Sayed Ali was born in Cairo, Egypt, in 1975. He is presently assistant professor of Organic Chemistry, Department of Chemistry, Faculty of Education, Ain Shams University, Cairo, Egypt. He graduated with B.Sc. (Physics and Chemistry) from Ain Shams University in 1997. He received his M.Sc. and Ph.D. degrees in 2001 and 2005, respectively, in Heterocyclic Chemistry from Ain Shams University. Awarded a post doctoral scientific grant for supporting young researchers (2007) from the Ministry of High Education and Scientific Research (Egypt) in organophosphorus laboratory, Institute of Polymers, Bulgarian Academy of Science, Sofia, Bulgaria. His CV was included in Who's Who in the World in 2011, 2012, 2013 and 2015. He won the award for the best research article in the field of Heterocyclic Chemistry at the Egyptian universities and research centers in 2011. He has published more than 40 scientific papers including 10 review articles, all in international journals. His research interests are in synthesis and chemical reactivity of phosphorus compounds containing bioactive heterocyclic systems.

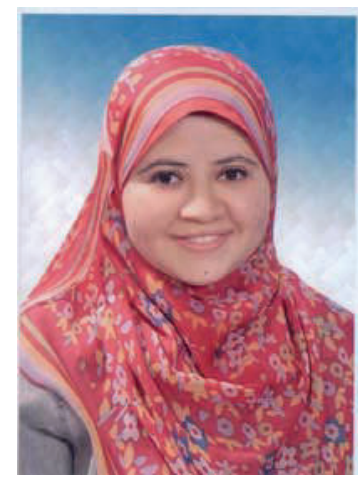

Somaia M. Abdel-Karim was born in 1985 in Cairo, Egypt. In 2006 she graduated from Ain Shams University, Faculty of Education, Department of Chemistry. Also, she received her M.Sc. and Ph.D. degrees in 2011 and 2014, respectively, in Organophosphorus Chemistry. She has published about 10 scientific papers, all in international journals. Her research interests are in synthesis and chemical reactivity of phosphorus compounds containing bioactive heterocyclic systems. 